\title{
Polymer Flow in Porous Media: Relevance to Enhanced Oil Recovery
}

\author{
Arne Skauge 1,2,*, Nematollah Zamani ${ }^{3}$, Jørgen Gausdal Jacobsen ${ }^{1,3}$, Behruz Shaker Shiran ${ }^{3}$, \\ Badar Al-Shakry 1 (iD) and Tormod Skauge ${ }^{2}$ \\ 1 Department of Chemistry, University of Bergen, Allegaten 41, N-5020 Bergen, Norway; \\ joja@norceresearch.no (J.G.J.); Badar.Al-Shakry@uni.no (B.A.-S.) \\ 2 Energy Research Norway, N-5020 Bergen, Norway; Tormod.Skauge@energyresearch.no \\ 3 Uni Research, N-5020 Bergen, Norway; Nematollah.zamani@uni.no (N.Z.); Behruz.shaker@uni.no (B.S.S.) \\ * Correspondence: arne.skauge@kj.uib.no; Tel.: +47-5558-3358
}

Received: 1 June 2018; Accepted: 3 July 2018; Published: 10 July 2018

\begin{abstract}
Polymer flooding is one of the most successful chemical EOR (enhanced oil recovery) methods, and is primarily implemented to accelerate oil production by sweep improvement. However, additional benefits have extended the utility of polymer flooding. During the last decade, it has been evaluated for use in an increasing number of fields, both offshore and onshore. This is a consequence of (1) improved polymer properties, which extend their use to HTHS (high temperature high salinity) conditions and (2) increased understanding of flow mechanisms such as those for heavy oil mobilization. A key requirement for studying polymer performance is the control and prediction of in-situ porous medium rheology. The first part of this paper reviews recent developments in polymer flow in porous medium, with a focus on polymer in-situ rheology and injectivity. The second part of this paper reports polymer flow experiments conducted using the most widely applied polymer for EOR processes, HPAM (partially hydrolyzed polyacrylamide). The experiments addressed highrate, near-wellbore behavior (radial flow), reservoir rate steady-state flow (linear flow) and the differences observed in terms of flow conditions. In addition, the impact of oil on polymer rheology was investigated and compared to single-phase polymer flow in Bentheimer sandstone rock material. Results show that the presence of oil leads to a reduction in apparent viscosity.
\end{abstract}

Keywords: EOR; polymer flooding; in-situ rheology; non-Newtonian flow in porous medium

\section{Introduction}

The success of polymer flooding depends on the ability of injected solutions to transport polymer molecules deep into a reservoir, thus providing enhanced mobility ratio conditions for the displacement process. In the following sections, we focus on the principal parameters that are crucial in the decision-making process for designing a satisfactory polymer flood design.

The application of polymer flooding to tertiary oil recovery may induce high injection pressures, resulting in injectivity impairment. Since the volumetric injection rate during polymer flooding is constrained by formation fracture pressure, project economics may be significantly affected. Thus, injectivity is a critical parameter and key risk factor for implementation of polymer flood projects.

A large number of injectivity studies, both theoretical and experimental, have been performed in porous media during recent decades, albeit they were mainly studies of linear cores in the absence of residual oil [1-7]. Recently, Skauge et al. [8] performed radial injectivity experiments showing significant reduction in differential pressure compared to linear core floods. This discrepancy in polymer flow in linear cores compared to that in radial disks is partly explained by the of differing pressure conditions that occur when polymer molecules are exposed to transient and semi-transient 
pressure conditions in radial disks, as opposed to the steady state conditions experienced in linear core floods. In addition, they observed that the onset of apparent shear thickening occurs at significantly higher flux in radial floods. Based on these results, injectivity was suggested to be underestimated from experiments performed in linear core plugs. However, these experiments were performed in the absence of residual oil. If residual oil has a significant effect on polymer propagation in porous media, experiments performed in its absence will not be able to accurately predict polymer performance.

Experimental studies investigating the effects of residual oil on polymer propagation through porous media have been sparse, although they have generally shown decreasing levels of polymer retention in the presence of residual oil $[9,10]$.

The polymer adsorbs to the rock surface and may also block pores due to polymer size (straining) and flow rate (hydrodynamic retention). In addition, different trapping mechanisms may take place. The polymer retention phenomena influence the flow of polymer in porous media, however, these effects are beyond the scope of this paper. The subject has been reviewed in several other books and papers, e.g., Sorbie [11] and Lake [12].

History matches performed in this study aim to highlight the injectivity of partially hydrolyzed polyacrylamides (HPAMs) in radial disks saturated with residual oil, as these conditions best mimic actual flow conditions in oil reservoirs. Results show that the presence of residual oil reduces the apparent viscosity of HPAM in flow through porous media, thus improving injectivity. These results may facilitate increased implementation of polymer EOR (enhanced oil recovery) projects, as previous projects deemed infeasible may now be economically viable.

\section{Theory}

\subsection{In-Situ Rheology}

Polymer viscosity as a function of shear rate is usually measured using a rheometer. During the measurement process, polymer solutions are exposed to different shear rates in a stepwise manner. For each shear rate, polymer viscosity is measured after steady state conditions are achieved; at this state, it is referred to as bulk viscosity. However, polymer molecules experience significantly different flow conditions in rheometers compared to porous media. In particular:

(I) unlike rheometers, porous media exhibit an inherently complex geometry;

(II) phenomena such as mechanical degradation may change rheological properties;

(III) although they only demonstrate shear thinning behavior in rheometers, polymer solutions may exhibit apparent shear thickening behavior above a certain critical flow rate;

(IV) due to the tortuosity of porous media and existence of several contraction-expansion channels, polymer solutions are exposed to a wide range of shear rates at each flow rate and where extensional viscosity becomes more dominant, resulting in significantly different rheology behavior compared to bulk flow.

To account for these contrasting flow conditions, in-situ viscosity has been suggested to describe the fluid flow behavior of polymer solutions in porous media. In-situ viscosity is a macroscopic parameter that can be calculated using Darcy's law for single-phase non-Newtonian fluids:

$$
\mu_{\text {app }}=\frac{K A}{Q} \frac{\Delta P}{L}
$$

It is generally measured in core flood experiments as a function of Darcy velocity. Comparison of in-situ and bulk rheology (Figure 1) shows vertical and horizontal shifts between viscosity curves. Vertical shifts may be due to phenomena such as mechanical degradation, while horizontal shifts are due to a conversion factor between in-situ shear rate and Darcy velocity, shown as $\alpha$. The red line in Figure 1 shows an increase in apparent viscosity, which is due to polymer adsorption. The adsorbed layer of polymer reduces the effective pore size and blocks smaller pores, both leading to increased 
resistance to flow e.g., as determined by an increase in pressure at a given rate compared to a non-adsorbing situation. In contrast, a reduction in pressure (and therefore, in apparent viscosity) can be observed in the presence of depleted layers (see e.g., Sorbie [11]) which leads to slip effects.

Due to the time-consuming nature of in-situ measurements, there have been several attempts to investigate in-situ rheology, both analytically and numerically. In spite of extensive studies [13-22], limited success has been achieved to reliably relate in-situ to bulk viscosity based on polymer solution and porous media properties. Most of these models were developed based on analytical solutions of non-Newtonian flow through capillary bundles, which simplifies the complex geometry of porous media.

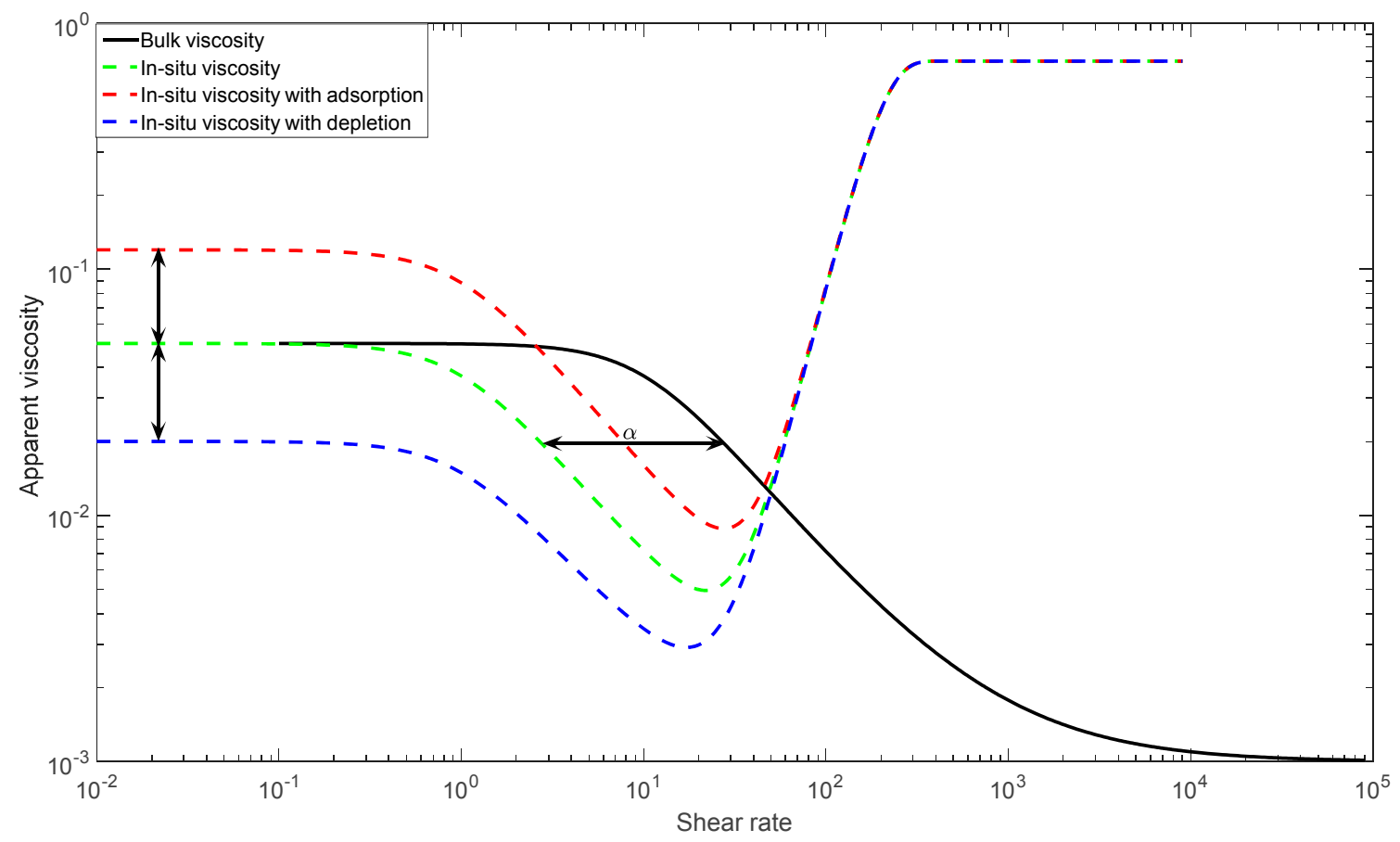

Figure 1. Schematic comparison of in-situ and bulk rheology.

In the following, the calculation procedure of in-situ viscosity is briefly explained:

1. Analytical solutions for a power-law fluid $\left(\mu=C \dot{\gamma}^{n-1}\right)$ at a given flow rate through a capillary tube with an arbitrary radius (R) can be defined by Equation (2). By comparing Equation (2) with the Poiseuille volumetric flow rate for Newtonian fluids in a tube (Equation (3)), an apparent viscosity and shear rate can be obtained from Equations (4) and (5), respectively.

2. The analytical equation in a single tube (Equation (5)) can be extended to account for real porous media by using the capillary bundle approach [23-25]. An equivalent radius of a capillary bundle model for porous media with known porosity $(\phi)$, permeability $(K)$ and tortuosity $(\psi)$ can be obtained by Equation (6). By calculating the Darcy velocity and substituting the equivalent radius (Equation (6)) into Equation (5), the apparent shear rate as a function of Darcy velocity can be obtained by Equation (7).

$$
\begin{gathered}
Q=\frac{\pi n}{3 n+1}\left(\frac{\Delta P}{2 C L}\right)^{1 / n} R^{\frac{3 n+1}{n}} \\
Q=\frac{\pi}{8 \mu} \frac{\Delta P}{L} R^{4} \\
\mu_{e f f}=C\left(\frac{3 n+1}{4 n}\right)\left(\frac{R \Delta P}{2 C L}\right)^{\frac{n-1}{n}}
\end{gathered}
$$




$$
\begin{gathered}
\mu_{a p p}=C \dot{\gamma}_{a p p}^{n-1} \Rightarrow \dot{\gamma}_{a p p}=\left(\frac{3 n+1}{4 n}\right)^{\frac{1}{n-1}}\left(\frac{R \Delta P}{2 C L}\right)^{1 / n} \\
R_{e q}=\sqrt{\frac{8 K \psi}{\phi}} \\
\dot{\gamma}_{a p p}=4\left(\frac{3 n+1}{4 n}\right)^{\frac{n}{n-1}} \frac{U}{\sqrt{8 K \phi \psi}}
\end{gathered}
$$

3. The above expressions are considered as an analytical basis for calculating apparent viscosity in porous media. Based on Equation (7), a simplified linear correlation between apparent shear rate and Darcy velocity is generally suggested, i.e., Equation (8), in which the correction factor $(\alpha)$ is the key factor. Some proposed equations for the correction factor are summarized in Table 1. By comparing different coefficients, different values for apparent viscosity may be obtained.

$$
\dot{\gamma}_{a p p}=\alpha \frac{U}{\sqrt{K \phi}}
$$

Table 1. Summary of proposed models for correction factor $(\alpha)$.

\begin{tabular}{ccc}
\hline Model & Equation for Correction Factor $(\alpha)$ & Description \\
\hline Analytical solution & $4\left(\frac{3 n+1}{4 n}\right)^{\frac{n}{n-1}}$ & $n$ is the power index in power-law region \\
Hirasaki and Pope [26] & $\frac{12}{\sqrt{150}}\left(\frac{3 n+1}{4 n}\right)^{\frac{n}{n-1}}$ & $n$ is the power index in power-law region \\
Cannella et al. [16] & $\frac{\beta}{\sqrt{S_{w}}}\left(\frac{3 n+1}{n}\right)^{\frac{n}{n-1}}$ & $n$ is the power index in power-law region, $S_{w}$ is \\
\hline
\end{tabular}

Based on the capillary bundle approach, other models were also proposed by Bird et al. [24], Christopher and Middleman [25], and Teeuw and Hesselink [15], in which the modified Blake-Kozeny model is used for power-law fluids (Equation (9)) and apparent viscosity is obtained using Equation (10).

$$
\begin{gathered}
U=\left(\frac{K}{\mu_{a p p}} \frac{\Delta P}{L}\right)^{1 / n} \\
\mu_{a p p}=C\left(\frac{3 n+1}{4 n}\right)^{n}\left(\frac{K \phi}{\beta}\right)^{(1-n) / 2}
\end{gathered}
$$

Based on the discussion given by Teeuw and Hesselink [15], tortuosity has a dual effect on both shear rate and shear stress calculations. Christopher and Middleman [25] only incorporated tortuosity in shear stress calculations, while Bird et al. [24] incorporated tortuosity into the shear rate term. The various values of $\beta$ chosen by different authors are summarized in Table 2.

Table 2. $\beta$ values applied by different authors where $\psi=25 / 12$.

\begin{tabular}{cc}
\hline Model & B \\
\hline Bird et al. [24] & $\sqrt{2 \psi}$ \\
Christopher and Middleman [25] & $\sqrt{\frac{2}{\psi}}$ \\
Teeuw and Hesselink [15] & $\sqrt{2}$ \\
\hline
\end{tabular}

Hirasaki and Pope [26] conducted several core flood experiments where permeability was in the range $7-23 \mathrm{mD}$, porosity in the range $18-20 \%$ and residual oil between $20 \%$ and $32 \%$. Based on these 
experiments, they concluded that apparent viscosity could be calculated using the capillary bundle approach and Blake-Kozeny model as follows:

$$
\mu_{a p p}=H U^{n-1}
$$

where:

$$
H=\frac{C}{12}\left(\frac{9 n+3}{n}\right)^{n}(150 K \phi)^{\frac{1-n}{2}}
$$

They also included pore size distribution in their calculations:

$$
\mu_{\text {app }}=\frac{C}{4}\left(\frac{1+3 n}{n}\right)^{n} \frac{\int_{0}^{\infty} \sigma(R) R^{2} d R}{\left[\int_{0}^{\infty} \sigma(R) R^{\frac{1+n}{n}} d R\right]^{n}}\left(\frac{q}{\phi}\right)^{n-1}
$$

Sadowski and Bird [16] used the Ellis model to obtain viscosity from the shear rate. The following equations for apparent viscosity were suggested based on the Blake-Kozeny model and capillary bundle approach:

$$
\begin{gathered}
\frac{1}{\mu_{e f f}}=\frac{1}{\mu_{0}}\left(1+\frac{4}{n+3}\left[\frac{\tau_{R H}}{\tau_{1 / 2}}\right]^{n-1}\right) \\
\tau_{R h}=\left(\frac{\Delta P}{L}\right)\left[\frac{D_{p} \phi}{6(1-\phi)}\right]
\end{gathered}
$$

In the above expressions, $\mu_{0}, \tau_{1 / 2}$ and $n$ are Ellis model parameters that can be measured in rheometers. By applying these equations, they obtained an acceptable match between experimental and predicted results for low to medium molecular weight polymers.

In summary, none of the proposed models for non-Newtonian fluids in porous media based on the capillary bundle approach are in agreement with all experimental results. Therefore, some known limitations of the capillary bundle approach are noted as follows:

- It neglects complex features of porous media such as tortuosity and pore size distribution.

- It assumes unidirectional flow as it neglects interconnectivity between pores.

- It cannot be representative for flow in an anisotropic medium due to its assumption of unique permeability along propagation direction.

- It assumes a single radius along bundles with no variation in cross-sectional area. The contraction-expansion feature of non-Newtonian flow in porous media is of high importance, especially when studying extensional viscosity, yield stress and elasticity.

- It is generally developed based on rheological models in which analytical solutions for velocity profiles are available (e.g., power-law and Ellis model). Analytical solutions for some models (e.g., Carreau model) are quite difficult and the equation for velocity is implicit (Equation (10) for the Carreau model) and needs to be solved iteratively.

$$
\frac{\partial v_{z}}{\partial r}=-\frac{\Delta p r}{2 L}\left\{\mu_{\infty}+\frac{\mu_{\infty}-\mu_{0}}{\left[1+\left(\lambda \frac{\partial v_{z}}{\partial r}\right)^{2}\right]^{\frac{n-1}{2}}}\right\}
$$

Duda et al. [27] studied polymer solution rheology inside porous media and reported that experimentally measured pressure drops were greater than those predicted by capillary bundle models, especially at lower values of the Carreau power index. Based on their study, a key reason for underestimating correction factors using the capillary bundle approach is the model's failure to capture either the interconnectivity of pores or non-uniform cross-sections of pore bodies and pore throats (i.e., abrupt contractions and expansions, also known as aspect ratio). 
According to the aforementioned limitations of the capillary bundle approach and lack of a universally accepted equation for calculating shear rates in porous media, the application of effective medium theory was eventually suggested. This method was able to remediate certain weaknesses in capillary bundle approach, for example, by incorporating pore interconnectivity and variation in cross-sections. Canella et al. [18] extended this method to account for power-law fluids in porous media. Core floods were conducted using xanthan in the concentration range 300-1600 ppm, rock lithology (sandstone and carbonates) in the permeability range $40-800 \mathrm{mD}$ and various oil residuals $(0-29 \%)$. Their general assumption was that bulk rheological properties of polymer solutions obey the power-law model, and they suggested the following equation for the relation between shear rate and Darcy velocity based on effective medium theory:

$$
\dot{\gamma}_{a p p}=\beta\left(\frac{3 n+1}{4 n}\right)^{\frac{n}{n-1}} \frac{q}{\sqrt{K \phi S_{w}}}
$$

Canella et al. achieved a satisfactory match with their experimental results by using a constant value of 6 for $\beta$, although this value far exceeds correction factors suggested by other researchers [28-30]. Even though all published results in the literature are not covered by using this correction factor, better agreement between analytical and experimental results was obtained, such as in experiments performed by Teeuw and Hesselink [15] and Gogarty [31].

Canella et al. [18] demonstrated that apparent viscosity depends on both microscopic (connectivity, pore size distribution) and macroscopic properties (permeability, porosity) of porous media. Despite calculation improvements, neither effective medium theory nor the capillary bundle model are able to accurately estimate the correction factor. The great discrepancies in results obtained by the models described above and the wide range of correction factors suggested [17] confirm that a universally accepted model does not yet exist. Insufficiency of these models to predict in-situ viscosity may be attributed to their lack of incorporating time dependence and their use of oversimplified porous media models (e.g., capillary bundle).

To avoid over-simplification of porous media obtained by using the capillary bundle approach, pore network modelling has been suggested. In contrast to the capillary bundle approach, pore network modeling envisages porous media as interconnected bundles with idealized geometries where larger pores (pore bodies) are connected via smaller ones (pore throats). Pore network models have been used by Sorbie et al. [20] to study non-Newtonian fluids that exhibit shear thinning properties; later, several authors studied these phenomena [21,32-35]. Using network modeling, Sorbie et al. [20] showed that in connected (2D) networks of porous media, the average shear rate in the network correlates linearly with the flow rate. This result is not obvious and indeed is rather unexpected. Thus, any formula of the form of Equation (8) which is linear in $U$, and has a "shift factor", will do well for shear thinning fluids. The paper also shows that a similar argument holds for extensional flow where the extensional rate in the porous medium correlates linearly with flow rate $(U)$. Lopez et al. [21] applied a pore network model to study non-Newtonian fluids using the same approach as for Newtonian fluids, except that viscosity in each bundle was not assumed to be constant and was considered as a function of pressure drop. Therefore, an iterative approach was suggested to calculate pressure drop and apparent viscosity. Although they obtained satisfactory agreement between analytical and experimental results using this approach, Balhoff and Thompson [34] stated that effects of concentration were neglected, and consequently proposed a new model based on CFD calculations to include effects of concentration in calculating conductivity of pore throats. They used pore network modeling to model shear thinning polymer flow with yield stress within a sand-pack.

Zamani et al. [35] studied the effects of rock microstructures on in-situ rheology using digital rock physics and reported that microscopic properties such as aspect ratio, coordination number and tortuosity may affect deviation of in-situ from bulk rheology.

In some experiments $[23,27,31,36]$, in-situ rheology has been reported to deviate significantly from the behavior in bulk flow, such that in-situ rheology may not be calculated directly from bulk 
rheology using the previously mentioned models. To achieve this, one may use these approaches assuming that either in-situ rheological properties are different from bulk rheological properties (e.g., Hejri et al. [36]) or that the relationship between apparent shear rate and Darcy velocity is non-linear (e.g., Gogarty [31]).

Calculation of in-situ rheology is a controversial subject. Until now, there has been no direct method to obtain it and, generally it has been measured by performing core floods. However, Skauge et al. [37] observed significantly different in-situ rheology for HPAM in linear compared to radial geometry. This discrepancy might be due to differing pressure regimes and flux conditions experienced by polymer solutions flowing through these inherently different flow geometries.

The problem with in-situ rheology calculations extends beyond finding the appropriate correction/shift factor. It also encompasses predicting the onset of extensional viscosity, which is treated as a separate subject in the following section.

\subsection{Extensional Viscosity}

Several experimental results show that, although polymer solutions (e.g., HPAM) only demonstrate shear thinning behavior in a rheometer, they may exhibit apparent shear thickening behavior above a critical shear rate in porous media (Figure 2) $[23,27,31,36]$. Generally, polymer flow in porous media may be divided into two distinct flow regimes: shear dominant and extensional dominant flow regimes. Since apparent shear thickening occurs in the extensional flow regime, it may also be referred to as extensional viscosity.

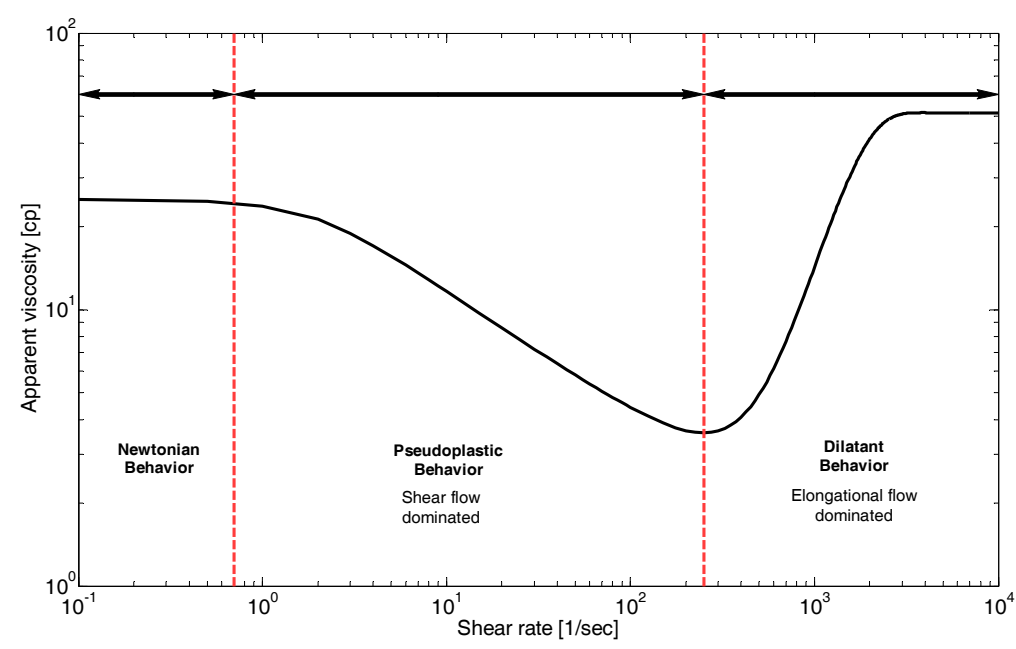

Figure 2. Schematic illustration of apparent viscosity in porous media.

Although its source is poorly understood, extensional viscosity is considered one of the principal aspects of polymer flow in porous media due to its influence on injectivity and oil mobilization. This phenomenon was suggested to be a consequence of elastic properties of polymer solutions (elongational dominated [38] or inertia-dominated flow [39]). As a result, extensional viscosity is often used interchangeably with elongational viscosity, shear thickening behavior, viscosity enhancement, dilatant behavior and viscoelasticity. Two different models are generally used to explain this phenomenon, the transient network model [40-42] and coil stretch model [43]. We adhere to the latter of these models.

Polymer molecules may be envisaged as entangled coils, and when exposed to a flow field, two forces may arise. First, an entropic force that attempts to maintain the existing polymer coil configuration. As coil entanglement increases, higher resistance to deformation is observed. Second the drag force resulting from interactions between solvent fluid and polymer molecules. When shear rate increases beyond a critical rate, molecule configurations change abruptly from coil to stretched states. Therefore, 
polymer coils start to deform, resulting in anisotropy and stress differences between elongation and compression. Consequently, normal stresses and elastic properties become more dominant.

Choplin and Sabatie [40] suggested that when polymer molecules are exposed to a simple shear flow at a constant shear rate $(\dot{\gamma})$, molecules rotate at a constant angular velocity $(\omega)$ proportional to applied shear rate, and in each rotation polymer molecules are stretched and compressed. The time between each rotation can be calculated by Equation (18).

$$
t=\frac{\pi}{2} k \dot{\gamma}
$$

where $k$ is a constant of proportionality, related to viscosity. If $t$ is higher than the Zimm relaxation time, no dilatant behavior occurs. Consequently, the critical shear rate at the onset of dilatant behavior may be calculated based on Zimm relaxation time as follows:

$$
\begin{gathered}
\lambda_{z}=\frac{6}{\pi^{2}} \frac{M_{w}}{R T}[\mu]_{0} \mu_{s} \\
t \leq \lambda_{z}, \dot{\gamma} \geq \gamma^{*} \stackrel{W e}{\Rightarrow} \dot{\gamma} \lambda_{z} W e^{*}=\lambda_{z} * \dot{\gamma}^{*}=\frac{\pi}{2} k
\end{gathered}
$$

Polymer viscosity behavior in extensional flow may be entirely different from its behavior in pure shear flow, i.e., polymer solution may show simultaneous shear thinning and extension thickening behavior. Theoretically, extensional viscosity can be calculated from Equation (21), where $\mathrm{N}_{1}$ is normal stress difference and $\dot{\epsilon}$ is stretch rate. The relative importance of extensional viscosity and shear viscosity is defined by a dimensionless parameter known as the Trouton ratio (Equation (22)), initially proposed by Trouton [44]. For non-Newtonian fluids (especially viscoelastic fluids), $\operatorname{Tr}$ can reach very large values, such as $10^{3}$ to $10^{4}$ (i.e., when polymer solution demonstrates shear thinning and extension thickening simultaneously).

$$
\begin{aligned}
\mu_{e} & =\frac{N_{1}}{\dot{\epsilon}} \\
T r & =\frac{\mu_{e}}{\mu_{s}}
\end{aligned}
$$

In Figure 2, the in-situ viscosity of viscoelastic polymers is depicted in both shear and extensional flow regime. At the onset of polymer flow, the generated hydrodynamic force from fluid flow (i.e., drag force) is below the threshold value in terms of overcoming entropic forces. Therefore, polymer configuration persists in a coil shape, and viscosity remains constant and equal to the zero-shear rate viscosity (upper Newtonian plateau). As flow rate increases, polymer molecules are exposed to larger drag forces that disentangle polymer coils and aligns them along the flow direction. This coil alignment reduces resistance to flow (i.e., induces viscosity reduction) and is referred to as shear thinning. When the orientation of polymer molecules is completely aligned, they will start to stretch at increasing flow rates. A change in the deformation of polymer molecules may cause normal stress differences. At low stretch rates $(\dot{\epsilon}), N_{1}$ is very low and by increasing the stretch rate, $N_{1}$ dramatically increases. In other words, beyond the critical shear rate $\left(\dot{\gamma}_{c}\right)$, instead of intramolecular interaction, intermolecular interactions will develop which generate amorphous structures much larger than average polymer chain dimensions $[28,45]$.

Within the extensional flow regime, the apparent viscosity generally reaches a maximum value, subsequently followed by a decreasing viscosity interval. This phenomenon may be interpreted as high viscoelastic stresses causing polymer rupture and chain halving, and it has been reported as being more severe in low-permeability porous media [46]. As molecular rupture occurs, new molecular weight distributions emerge (larger molecular weight fractions are distorted) and viscosity behavior of the polymer may be governed by a new molecular weight distribution.

The onset of extensional viscosity-the transition point between shear-dominant and extensional dominant flow-depends on polymer, solvent, and porous media properties. The effects of polymer 
properties on extensional viscosity can be investigated by using special rheometers that only generate pure extensional flow [47-56]. In the following, the effects of polymer, solvent and porous media properties on the onset of extensional viscosity are explained.

\subsubsection{Polymer Concentration}

Chauveteau [55] reported that the maximum relaxation time increases with polymer concentration, thus dilatant behavior commences at lower shear rates (Figure 3). He also included the effect of concentration in the expression for Zimm relaxation time, producing Equation (23).

$$
\lambda_{z}=\frac{6}{\pi^{2}} \mu_{s} \frac{\mu_{r o}-1}{C} \frac{M_{w}}{R T}
$$

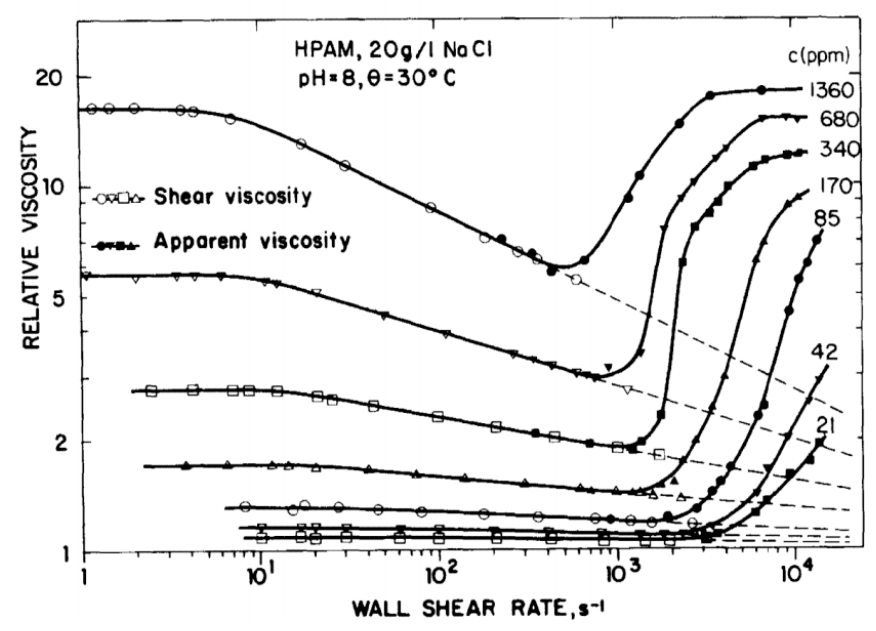

Figure 3. Effect of polymer concentration on the onset of extensional viscosity in a model with 45 successive constrictions. Reproduced with permission from [55].

The effect of concentration on extensional viscosity was also investigated by Lewandowska [56]. In contrast to Chauveteau, he reported that dilatant behavior commences at higher shear rates with increasing polymer concentration. He attributed this observation to the higher degree of entanglement as the concentration increases, thus increasing the extent of the shear thinning region.

Briscoe et al. [57] could not identify a consistent trend between polymer concentration and onset of extensional viscosity. They assumed that only a narrow region of polymer concentrations is able to generate apparent shear thickening behavior. Below a critical concentration limit, defined as the critical overlap concentration $\left(C^{*}\right)$, few polymer chains are able to form transient networks. At concentrations above $\mathrm{C}^{*}$, the extent of shear thinning may increase and, consequently, the onset of apparent shear thickening may be delayed. This effect was also studied by Dupuis et al. [58], where they observed that the onset of dilatant behavior decreased with polymer concentration. However, rheological behavior above the critical shear rate deviated among different concentration ranges (low: 30-60 ppm; medium: 120-240 ppm; and high: 480-960 ppm). Jiang et al. [59] also confirmed scattered data for the onset of extensional viscosity as function of polymer concentration. Clarke et al. [60] reported that the onset of extensional viscosity is independent of concentration and only depends on molecular weight.

\subsubsection{Molecular Weight}

The lengths of polymer chains increase with molecular weight, resulting in higher inter- and intramolecular entanglement. Thus, the extent of the shear thinning region increases and, consequently, delay the onset of dilatant behavior [56]. However, this explanation directly contradicts the expression for the Zimm relaxation time (Equation (23)), where the latter increases with molecular weight and causes critical shear rate to occur at a lower shear rate. 
Jiang et al. [59] also studied the effects of molecular weight on the onset of extensional viscosity. They concluded that relaxation time increases with molecular weight, thus the onset of extensional viscosity occurs at lower shear rates. In addition, they observed that this trend was not valid above a critical molecular weight.

Clarke et al. [60] proposed the following correlation for the dependency of the onset of extensional viscosity on polymer molecular weight:

$$
\lambda_{\text {ext }} \propto M W^{2} C_{p}^{0}
$$

\subsubsection{Salinity Effect}

The effect of salinity on polymer rheology may be crucial in some reservoir conditions [11,61,62], and depends on polymer type. For typical EOR polymers (e.g., xanthan, HPAM, or generally non-hydrolyzed polymers), increasing salinity generally reduces coil gyration and hydrodynamic radius. Due to the repulsion between ionic groups in HPAM solutions, increasing salinity compresses the electrical double layer on molecular chains and electrostatic repulsion decreases. In the case of HPAM, the reaction mechanism varies for different metal ions i.e., either monovalent $\left(\mathrm{Na}^{+}\right)$or divalent $\left(\mathrm{Ca}^{2+}\right)$ cations. In the monovalent case, it may suppress the charge effect and reduce the hydrodynamic radius. In the divalent case, reactions between cations (i.e., $\mathrm{Ca}^{2+}$ ) can play the role of cross-linkers and influence the conformation and rheological properties of HPAM. In both cases, larger shear rates are required to uncoil polymers and the apparent shear thickening commences at larger shear rates $[57,58,63]$.

\subsubsection{Degree of Hydrolysis}

When HPAM is dissolved in water, electrostatic repulsion forces cause polymer molecules to expand easily and the shear thinning region is shortened. Therefore, as the degree of hydrolysis increases, the onset of apparent shear thickening decreases [56].

\subsubsection{Pressure and Temperature Effect}

Although polymers are considered incompressible fluids, they do exhibit some degree of compressibility. Thus, pressure may have an impact on viscosity. By increasing pressure, the free volume between polymer molecules decreases and Brownian motion of polymer chains is inhibited, consequently resulting in viscosity increase of polymer solution. Experimental results [64] indicate that the onset of extensional viscosity decrease significantly with pressure.

The effects of temperature on polymer rheology has also been studied extensively $[57,59,65,66]$ and results show that the critical shear rate and onset of dilatant behavior are retarded with increasing temperature. This behavior may have the following two explanations. Firstly, polymer relaxation time and solvent viscosity should both decrease with increasing temperature, based on Equation (23). Secondly, solvent quality decreases with temperature. By decreasing solvent quality, coil size is reduced, and to compensate for this reduction, a larger shear rate is needed to uncoil and elongate the polymer. Therefore, the onset of extensional viscosity occurs at higher shear rates.

\subsubsection{Porous Media Properties}

In addition to polymer properties, porous media may also significantly influence the generation of extensional flow, as shown by several experimental [25] and numerical studies [67]. Due to variation in cross-sectional area along its propagation path, polymer molecules are forced to accelerate and decelerate. Consequently, they will experience both stretch and shear flow in porous media, and above a critical flow rate, extensional flow will dominate shear flow.

To envisage polymer flow in porous media, the latter may be considered as a simplified contraction-expansion channel. As polymer molecules enter contractions, they will be compressed and stretched. If the flow is below a critical velocity, deformed polymer molecules have sufficient time to 
return to their original state. Therefore, when polymer solutions enter subsequent contractions, no stress is stored and no additional resistance to flow is observed. However, if polymer relaxation time is high and polymer molecules are not able to return to their equilibrium state between contractions, stress will be stored and accumulated, thus resulting in steep increases in pressure drop and apparent viscosity. This phenomenon can be interpreted as a memory effect of polymer molecules.

Due to the inherent nature of porous media, polymer molecules are sheared near the wall and elongated at the flow axis. Therefore, molecular momentum is transferred by both tangential and normal stress components in porous media. Seeing that polymer molecules are able to rotate in pore space, molecules are not strained and effective viscosity is only controlled by shear. In contrast, if molecules are exposed to strain for sufficient time, molecule deformation plays a major role and effective viscosity will be defined by strain $[25,67-72]$.

To predict the onset of extensional viscosity in porous media, the dimensionless Deborah number is defined as a ratio between the characteristic relaxation time of a fluid $\left(\theta_{f}\right)$ and characteristic time of porous media $\left(\theta_{p}\right)$, considered as the average time to travel from one pore body to another (Equation (25)). In other words, the Deborah number may be interpreted as the ratio between elastic and viscous forces. Based on this expression, the Deborah number is zero for Newtonian fluids and infinity for Hookean elastic solids.

$$
N_{D e}=\frac{\theta_{f}}{\theta_{p}}
$$

Polymer solutions may have a wide range of molecular weights leading to a large number of relaxation times. Many researchers have used the longest relaxation time as representative of $\theta_{f}$. However, this may cause the overestimation of Deborah numbers at the onset of extensional viscosity. Relaxation times may also be calculated from normal stress differences [73].

Some experimental observations revealed that the onset of extensional viscosity occurs when $N_{D e}$ is larger than 0.5 [74]. However, the Deborah number is not constant in different experiments and a wide range of values has been reported. Marshall and Metzner [73] reported a Deborah number of 0.1 at the onset of extensional viscosity, while Chauveteau [55] reported a relatively high Deborah number of 10. This wide range of reported Deborah numbers at the onset of extensional viscosity is due to difficulties in calculating stretch rates in porous media. To support this idea, Heemskerk et al. [75] reported that by using different polymer types in the same rock sample, critical Deborah numbers $\left(N_{D e}\right)$ were identical. However, when the same polymer was used in different rock samples, the critical $N_{D e}$ varied between 1 and 2 . They concluded that measured relaxation times from experimental results can be used to practically define the onset of extensional viscosity, but they acknowledged that equations for calculating stretch rate are not able to capture the exact $N_{D e}$ at the onset of extensional viscosity. Zamani et al. [67] proposed that to obtain a more accurate estimation of the critical $N_{D e}$, the stretch rate distribution at the pore scale is required. Metzner et al. [76] concluded that the critical Deborah number might only be used as a first estimation of the onset of extensional viscosity. In Table 3, some suggested equations for the calculation of Deborah number are summarized.

Table 3. Proposed equations for Deborah number calculation.

\begin{tabular}{|c|c|c|}
\hline Model & Equation & Description \\
\hline Masuda et al. [77] & $\begin{array}{c}N_{D e}=\theta_{f} \dot{\gamma}_{e q} \\
\dot{\gamma}_{e q}=\frac{\dot{\gamma}_{c}\left|U_{w}\right|}{\sqrt{\bar{k} k_{r w} \phi S_{w}}}\end{array}$ & $\begin{array}{l}\text { They used the inverse of the shear rate for } \theta_{p} . U_{w} \text { is the } \\
\text { Darcy velocity, } k_{r w} \text { is the water relative permeability, } S_{w} \\
\text { is water saturation and } \dot{\gamma}_{c} \text { is a constant equal to } 3.97 C \text {, } \\
\text { where } C \text { is an empirical correlation factor to account for } \\
\text { the difference between an equivalent capillary model } \\
\text { and real porous media }\end{array}$ \\
\hline $\begin{array}{c}\text { Hirasaki and Pope [26] } \\
\text { Haas and Durst [78] } \\
\text { Heemskerk et al. [75] }\end{array}$ & $\begin{array}{c}\frac{1}{\theta_{p}}=\dot{\varepsilon}=\frac{v}{d}= \\
\frac{U_{w}}{\left(1-\phi S_{w}\right) \sqrt{150 K K_{r} /\left(\phi S_{w}\right)}}\end{array}$ & \\
\hline
\end{tabular}


Several experimental results $[68,79]$ show that the Deborah number alone is not sufficient to predict the onset of extensional viscosity. As an explanation, Ranjbar et al. [80] stated that the onset of extensional viscosity highly depends on the elastic properties of polymer solutions and relaxation time alone cannot capture viscoelastic properties. Experimental results reported by Garrouch and Gharbi [79] support this idea. They investigated two different polymer solutions (xanthan and HPAM) in Berea and sand-packs. Calculated Deborah numbers for these two completely and inherently different polymer solutions inside sand-packs were (surprisingly) identical. While xanthan consists of rigid, rod-like molecules that do not show extensional viscosity, HPAM consists of flexible and elastic chain-structured molecules.

Zamani et al. [67] numerically studied the effect of porous media on the onset of extensional viscosity by using real images of porous media obtained from digital rock physics. They confirmed that microscopic features of porous media had significant impact on the onset of extensional viscosity. Furthermore, by increasing the aspect ratio and inaccessible pore volume and decreasing the coordination number, extensional viscosity occurred at lower shear rates, in agreement with several experimental results $[55,68,81]$.

Skauge et al. [37] reported that in radial flow, the onset of extensional viscosity occurred at higher shear rates than at typical core flooding. Since radial flow is more representative of real field conditions, results obtained from radial disks should be more accurate as laboratory data for field implementation.

Briefly summarized, at low shear rates where the amplitude of the elastic component is negligible, flow is controlled by shear forces. In contrast, above a critical shear rate, flow is extensional and governed by elastic forces. Therefore, the response of polymer solutions to imposed stress may be expressed as the sum of shear and elastic components:

$$
\begin{gathered}
\Delta P=\Delta P_{\text {shear }}+\Delta P_{\text {elastic }} \\
\mu=\mu_{\text {shear }}+\mu_{\text {elastic }}
\end{gathered}
$$

The viscosity of polymer solutions under shear flow can be described by empirical equations such as the power-law and Carreau models. To describe viscosity under elongational flow, several models have been suggested, and some of them are summarized in Table 4.

\begin{tabular}{|c|c|c|}
\hline Model & Equation & Description \\
\hline Hirasaki and Pope [26] & $\mu_{e l}=\frac{\mu_{s h}}{\left[1-N_{D e}\right]}$ & \\
\hline Masuda et al. [77] & $\mu_{\text {elas }}=\mu_{s h} C_{c}\left(N_{D e}\right)^{m_{c}}$ & where $C_{c}$ and $m_{c}$ are constant and relate to pore geometry \\
\hline Delshad et al. [61] & $\begin{array}{c}\mu_{e l}=\mu_{\max }\left[1-\exp \left(-\left(\lambda_{2} \tau_{r} \dot{\gamma}\right)^{n_{2}-1}\right)\right] \\
\tau_{r}=\tau_{1}+\tau_{0} C_{p} \\
\mu_{\max }=\mu_{w}\left(A P_{11}+A P_{22} \ln C_{p}\right)\end{array}$ & $\begin{array}{l}\tau_{r} \text { is the characteristic relaxation time and can be } \\
\text { calculated by dynamic frequency sweep test in the } \\
\text { laboratory. Some empirical correlations are also proposed } \\
\text { for dependency of different parameters on polymer } \\
\text { concentration }\end{array}$ \\
\hline Stavland et al. [62] & $\begin{array}{c}\mu_{e l}=\left(\lambda_{2} \dot{\gamma}\right)^{m} \\
\lambda_{2}=\left\{N_{D e}\left(\frac{1-\phi}{\phi}\right)\left(\frac{6 \alpha \sqrt{\tau}}{\lambda_{1}}\right)\right\}^{-1}\end{array}$ & $\begin{array}{l}m \text { is a non-zero tuning parameter which is known as the } \\
\text { elongation exponent and depends on the molecular } \\
\text { weight and demonstrates linear correlation with }[\mu] C_{p} . \alpha \\
\text { in the listed formulation is considered } 2.5\end{array}$ \\
\hline
\end{tabular}

Table 4. Proposed models for calculation of elongational viscosity.

\subsection{Injectivity}

Polymer injectivity is a crucial factor governing the economics of polymer flooding projects and its accurate estimation is a prerequisite in terms of optimizing the upper-limit injection rate [82]. Injection well pressure may increase due to one of the following causes: (1) oil bank formation, (2) in-situ polymer viscosity (especially shear thickening due to viscoelasticity) and (3) different types of retention, which cause permeability reduction. 
The highest pressure drops observed during polymer flooding are located in the vicinity of the injection wellbore due to dramatic variations in flow rate. Therefore, it is important to include non-Newtonian effects of polymer solutions to accurately predict polymer injectivity. Although both HPAM and xanthan demonstrate shear thinning behavior at low to moderate shear rates, HPAM exhibits apparent shear thickening above a critical flow rate due to its inherent viscoelastic nature. For field applications, injection rates in the vicinity of the injection well may easily exceed the onset of extensional viscosity, and injectivity will then dramatically decrease. In contrast to HPAM, xanthan shows exclusively shear thinning behavior and will attain its highest value of injectivity in the near-wellbore region.

Injectivity investigations at the lab scale are required before implementing field applications, and effects of polymer solution properties, in-situ rheology, temperature, $\mathrm{pH}$, level of retention and the nature of porous media should be accurately measured [83,84]. Furthermore, if screening criteria for polymer type are disregarded, polymer entrapment in narrow pore throats can have significant effects on its injection rate. The salinity of solutions can also affect polymer solubility, resulting in filter cake formation near injection wells or precipitation of polymer molecules in the reservoir. Inaccurate measurement of in-situ rheology and especially the onset of extensional viscosity may lead to either an underestimation or overestimation of injectivity. In some polymer flooding projects, measured injectivity may differ significantly from the simulation or analytical forecast. These unexpected injectivities may be due to the occurrence of mechanical degradation $[82,85,86]$, induced fractures [87-89], or even inaccurate analytical models for calculating in-situ rheology and predicting extensional viscosity.

\section{Radial In-Situ Rheology}

Injectivity (I) may be defined as the ratio of volumetric injection rate, $Q$, to the pressure drop, $\Delta P$, associated with polymer propagation between injection well and producer [1]:

$$
I=\frac{Q}{\Delta P}
$$

As previously mentioned, formation fracture pressure may constrain the value of volumetric injection rate. Due to its significant effect on project economics, accurate determination of differential pressure, and hence injectivity, at a given injection rate is essential. To achieve this, all factors affecting differential pressure during polymer flooding must be quantified. Darcy's law for radial flow may be expressed in terms of differential pressure as follows:

$$
\Delta P=\frac{\mu_{a p p} Q}{2 \pi h k_{e, i}} \ln \frac{r_{e}}{r_{w}}
$$

where $\mu_{a p p}$ is apparent viscosity, $h$ is disk thickness, $k_{e, i}$ is effective permeability to polymer solution, $r_{e}$ is disk radius and $r_{w}$ is injection well radius.

In this paper, the ratio of resistance factor $(R F)$ to residual resistance factor $(R R F)$ is used to represent apparent viscosity of polymer solutions propagating through porous media, thus isolating its viscous behavior, i.e.,

$$
\mu_{a p p}=\frac{R F}{R R F}
$$

where the resistance factor $(R F)$ represents the pressure increase of polymer relative to brine and the residual resistance factor $(R R F)$ is defined as the ratio of pressure before and after polymer injection (i.e., pressure caused by irreversible permeability reduction induced by retention mechanisms).

Due to their inherent viscoelastic behavior in porous media, synthetic polymers (e.g., HPAM) will exhibit shear-dependent apparent viscosity. Although the common consensus on apparent shear thickening as a phenomenon is accepted, its viscosifying magnitude is still an ongoing topic of debate in scientific communities. 
Accurate polymer rheology estimation is a prerequisite for reasonable injectivity estimates due to the proportionality between apparent viscosity and differential pressure. In linear core floods where steady-state pressure conditions exist, polymer flux will remain constant from inlet to outlet, rendering rheology estimation a straightforward task. However, in radial flow, polymer flux is gradually reduced as it propagates from injection well to producer, therefore attaining a range of viscosities rather than one specific value. Since the degree of mechanical degradation generally increases with injection rate, discrepancies in polymer rheology obtained from different injection rates may transpire. Instead of possessing one definite rheology, polymers propagating through radial disks will exhibit both shear-dependent and history-dependent viscosity behavior, thus increasing the complexity of rheology estimation in radial compared to linear models. To date, no correction factor has been suggested to account for this dual nature phenomenon. Even when mechanical degradation is excluded, i.e., when injected and effluent viscosities are approximately equal, this dual nature phenomenon persists, and is suggested to be attributed to non-equilibrium pressure conditions experienced in radial flow and inherent history-dependent nature of polymer molecules.

In addition, synthetic polymers are susceptible to mechanical degradation at high flux, typically in the near-wellbore region, which will impart an irreversible viscosity reduction due to polymer molecule fragmentation. Mechanical degradation induces a pressure drop that improves injectivity. However, since it disrupts the carefully selected viscous properties of the polymer solutions by a non-reversible viscosity decrease, mechanical degradation is not a sought-after phenomenon in polymer flooding. A remediation measure to reduce mechanical degradation is to pre-shear the polymer before injection. Pre-shearing removes the high molecular weight part of the molecular weight distribution, which is believed to be most susceptible to mechanical degradation [6]. Mechanical degradation may also be minimized by shifting to a lower molecular weight polymer. However, this would require higher amounts of polymer to obtain the same concentration, thus potentially influencing polymer project economics.

As mentioned, in radial geometry, high flux causing mechanical degradation occurs principally in the near-wellbore region, as opposed to linear geometry where this high flux persists throughout the entire propagation distance. Therefore, the time that polymer is exposed to high shear is short in radial transient flow pattern, as opposed to that of a steady-state linear core flood, [34]. Based on this time-differing condition between linear and radial flow, it was suggested by Skauge et al. that polymer is degraded to a lesser extent in radial compared to linear flood when injected at the same volumetric flow rate [33].

In summary, there are two principal factors governing injectivity during polymer flooding in linear geometry: (1) viscoelasticity of polymer that induces large injection pressures mainly due to apparent shear thickening behavior at high flux; and (2) mechanical degradation in the near-wellbore region, which causes an entrance pressure drop [1]. In radial disks, two additional factors should be included: (3) non-equilibrium pressure conditions due to kinetic effects; and (4) memory-effects of polymer molecules in non-constant velocity fields.

\section{Materials and Methods}

Rock: Bentheimer outcrop rock (porosity of $\sim 23 \%$, permeability of about 2.6 Darcy). Based on XRD measurements, Bentheimer consists predominantly of quartz $(90.6 \%)$ with some feldspar $(4.6 \%)$, mica $(3.2 \%)$ and siderite $(1.0 \%)$.

Polymer: Flopaam 3630S, 30\% hydrolyzed, MW = 18 million Da.

Brine: Relatively low salinity with a low content of divalent ions. Brine composition by ions is given in Table 5. 
Table 5. Brine ionic composition.

\begin{tabular}{cc}
\hline Ion & Concentration $(\mathbf{p p m}, \boldsymbol{w} / \boldsymbol{w})$ \\
\hline $\mathrm{Na}$ & 1741 \\
$\mathrm{~K}$ & 28 \\
$\mathrm{Ca}$ & 26 \\
$\mathrm{Mg}$ & 17 \\
$\mathrm{SO}_{4}$ & 160 \\
$\mathrm{Cl}$ & 2687 \\
$\mathrm{TDS}$ & 4659 \\
Ionic strength & 0.082 \\
Hardness & 43 \\
\hline
\end{tabular}

Linear core floods: Core data are summarized in Table 6. All experiments were performed at room temperature and pressure.

Radial core floods: Bentheimer disks were prepared by coating with epoxy resin, vacuuming and saturating with brine. One disk was then drained with an extra heavy oil and aged for 3 weeks at $50{ }^{\circ} \mathrm{C}$ to a non-water-wet state. The crude had an initial viscosity of about $7000 \mathrm{cP}$. The extra heavy oil used for drainage and aging, was then exchanged with a flooding oil of $210 \mathrm{cP}$. Both experiments were performed at room temperature and pressure. Core data are given in Table 7 . The pressure ports were located in the injection and production wells and at radii $0.5,1.0,1.5,2.0,3.0,4.0$ and $5.0 \mathrm{~cm}$ for the disk without oil and at radii 1.1,2.0, and $5.0 \mathrm{~cm}$ for the disk containing oil.

The Bentheimer cores show a pore-throat distribution function similar to other outcrop sandstone material, Figure 4. All porous media have local pore-size variation, involving continuous contraction and expansion of pore-scale transport.

Table 6. Core data for linear core floods.

\begin{tabular}{cccccccccc}
\hline Experiment & Conc. & $\mathbf{L}(\mathbf{c m})$ & $\mathbf{D}(\mathbf{c m})$ & $\boldsymbol{\phi}(-)$ & $\mathbf{K}_{\mathbf{w i}}$ (Darcy) & $\mathbf{K}_{\mathbf{w f}}($ Darcy) & $\mathbf{R R F}(-)$ & $\eta_{\mathbf{i}}(\mathbf{c P})$ & $\eta_{\mathbf{e}}(\mathbf{c P})$ \\
\hline No oil & $500 \mathrm{ppm}$ & 9.54 & 3.77 & 0.24 & 2.48 & 1.35 & 1.84 & 6.81 & 6.62 \\
No oil & $1500 \mathrm{ppm}$ & 4.89 & 3.79 & 0.24 & 1.99 & 0.32 & 6.29 & 33.76 & 32.87 \\
With oil, not aged & $500 \mathrm{ppm}$ & 10.44 & 3.78 & 0.23 & 1.83 & 0.36 & 5.08 & 6.65 & 6.77 \\
With oil, aged & $500 \mathrm{ppm}$ & 9.85 & 3.78 & 0.23 & 2.27 & 0.27 & 8.41 & 6.99 & 5.90 \\
\hline
\end{tabular}

Table 7. Core data for radial core floods.

\begin{tabular}{ccccccccccc}
\hline Experiment & $\begin{array}{c}\text { Diameter } \\
(\mathbf{c m})\end{array}$ & $\begin{array}{c}\text { Thickness } \\
(\mathbf{c m})\end{array}$ & $\begin{array}{c}\text { Well Radius } \\
\mathbf{( \mathbf { c m } )}\end{array}$ & $\begin{array}{c}\phi \\
(-)\end{array}$ & $\begin{array}{c}\mathbf{P V} \\
(\mathbf{m L})\end{array}$ & $\begin{array}{c}\text { Soi } \\
(\mathbf{f r a c})\end{array}$ & $\begin{array}{c}\text { Sorw } \\
(\mathbf{f r a c})\end{array}$ & $\begin{array}{c}\mathbf{K}_{\mathbf{w}, \mathbf{a b s}} \\
(\mathbf{D a r c y})\end{array}$ & $\begin{array}{c}\mathbf{K}_{\mathbf{w}, \text { Sorw }} \\
(\mathbf{D a r c y})\end{array}$ & $\begin{array}{c}\mathbf{K}_{\mathbf{w f}} \\
(\mathbf{D a r c y})\end{array}$ \\
\hline No oil & 30.00 & 2.20 & 0.15 & 0.24 & 373 & n.a. & n.a. & 2.600 & n.a. & 0.056 \\
With oil & 29.90 & 2.21 & 0.30 & 0.23 & 352 & 0.91 & 0.22 & 1.551 & 0.041 & 0.039 \\
\hline
\end{tabular}

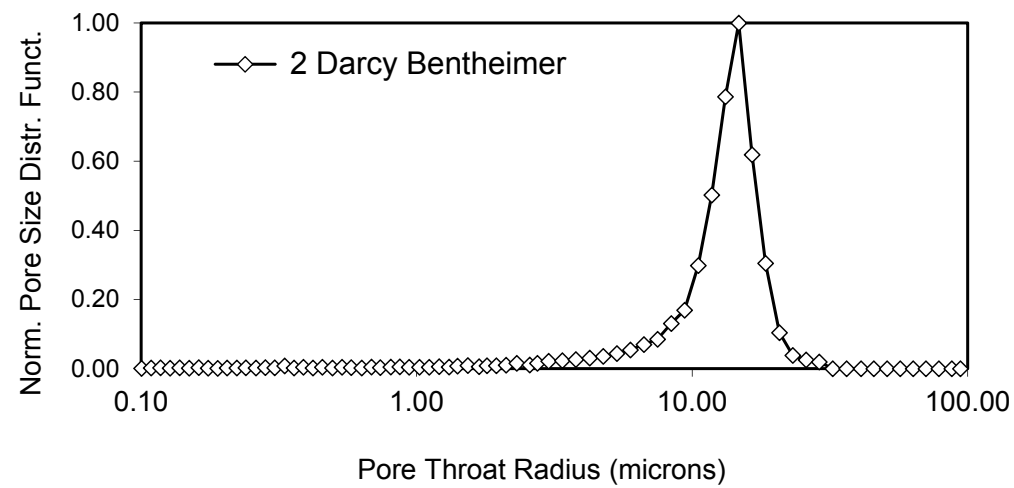

Figure 4. Mercury injection derived pore throat distribution for Bentheimer core material used in the polymer flow experiments. 
Simulation: The experimental set-up enabled detailed monitoring of pressure by internal pressure ports located at various distance from the injection well. Differential pressure as function of radial distance was history matched using the STARS simulator, developed by Computer Modeling Group (CMG). The simulation model encompassed a radial grid with 360 sectors, each consisting of 150 grid block cells in radial direction, where the grid block cell size is $1 \mathrm{~mm}$. Porous media permeability (tuning parameter) was obtained by history matching water floods prior to polymer flooding. Local permeability variation improved the history match compared to analytical solution (Darcy's law for radial flow). Permeability data obtained from water floods were used in subsequent polymer floods to isolate the effects of polymer apparent viscosity on differential pressure. In polymer floods, as the permeability obtained from the precursor water flood was held constant, apparent viscosity could be quantitatively investigated as a function of velocity and was used as the tuning parameter to history match differential pressure. The STARS simulation tool can include both shear thinning and thickening behavior of viscoelastic fluids.

Due to the inherent grid averaging calculation method of the simulation tool, the velocity in the first grid block after the injection well was below its analytical value. Because of a rapid velocity decrease with distance in radial models, this phenomenon was addressed by decreasing the injection well radius, thus effectively parallel shifting the position of the first grid block towards the injection well until the correct velocity was attained. This was a necessary step, since the tuning parameter is apparent viscosity as a function of velocity.

\section{Polymer In-Situ Rheology in Linear Cores}

Four Bentheimer outcrop cores were used to study polymer in-situ rheology in linear systems. Petro-physical properties of core samples as well as properties of polymer solutions are given in Table 6. Two experiments were carried out to examine the effect of polymer concentration on in-situ rheology of the polymer solution. Partially-hydrolyzed Flopaam $3630 \mathrm{~S}$ at $500 \mathrm{ppm}$ and $1500 \mathrm{ppm}$ was injected into the cores and the in-situ rheology of the polymer solutions was measured. The two concentrations were chosen to give viscosities representative of the upper and lower limit of what would be economically viable for polymer flooding in an oil field. Both concentrations are above the polymer critical overlap concentration, $C^{*}$. The results are presented in Table 6 and Figure 5. The bulk viscosity of $1500 \mathrm{ppm} 3630 \mathrm{~S}$ is about $34 \mathrm{cP}$ which is about 5 times that of $500 \mathrm{ppm} 3630 \mathrm{~S}$. Comparing in-situ rheology of $500 \mathrm{ppm}$ and $1500 \mathrm{ppm} 3630 \mathrm{~S}$ shows that the onset and degree of apparent shear thickening behavior are fairly similar for both concentrations. This is in line with observations by Skauge et al. [8] and Clarke et al. [60] that the onset of extensional viscosity is independent of polymer concentration and only depends on polymer molecular weight. It is noted that this is generally only true for $\mathrm{C}^{*}<\mathrm{C}<\mathrm{C}_{\text {lim }}$, where $\mathrm{C}_{\text {lim }}$ is the economic limit for polymer concentration, typically between 1500 and 2500 ppm. Table 6 and Figure 5 show that the magnitude of resistance factor (RF) and residual resistance factor (RRF) are about 4 and 3 times higher for $1500 \mathrm{ppm}$ compared to $500 \mathrm{ppm}$, respectively. This implies that polymer injectivity is a function of polymer concentration, and better injectivity is achieved with lower polymer concentrations.

A series of experiments was also performed to study the effect of the presence of residual oil on polymer in-situ rheology. In these experiments, Bentheimer cores at residual oil saturations of about $22 \%$ and different initial wettability states were flooded with polymer and the in-situ rheology behavior was compared to that of single-phase polymer injection in absence of residual oil. Prior to polymer injection, the cores containing oil were water flooded to residual oil saturation. At the end of the water flood, the flow rates were increased to generate pressures higher than that expected for the subsequent polymer flood. This was performed in order to avoid oil mobilization during the polymer flood and, indeed, no oil production was observed during the subsequent polymer flood. The results are presented and compared in Figure 6. As this figure shows, the onset of apparent shear thickening is not affected by the presence of residual oil or the wettability state of the cores. However, the slope of apparent shear thickening and magnitude of resistance factor is significantly affected by oil presence in 
the cores. That is, although onset of apparent shear thickening is independent of oil presence in porous media and its wettability condition, the results show that the degree of apparent shear thickening is lower when oil is present in the porous media.

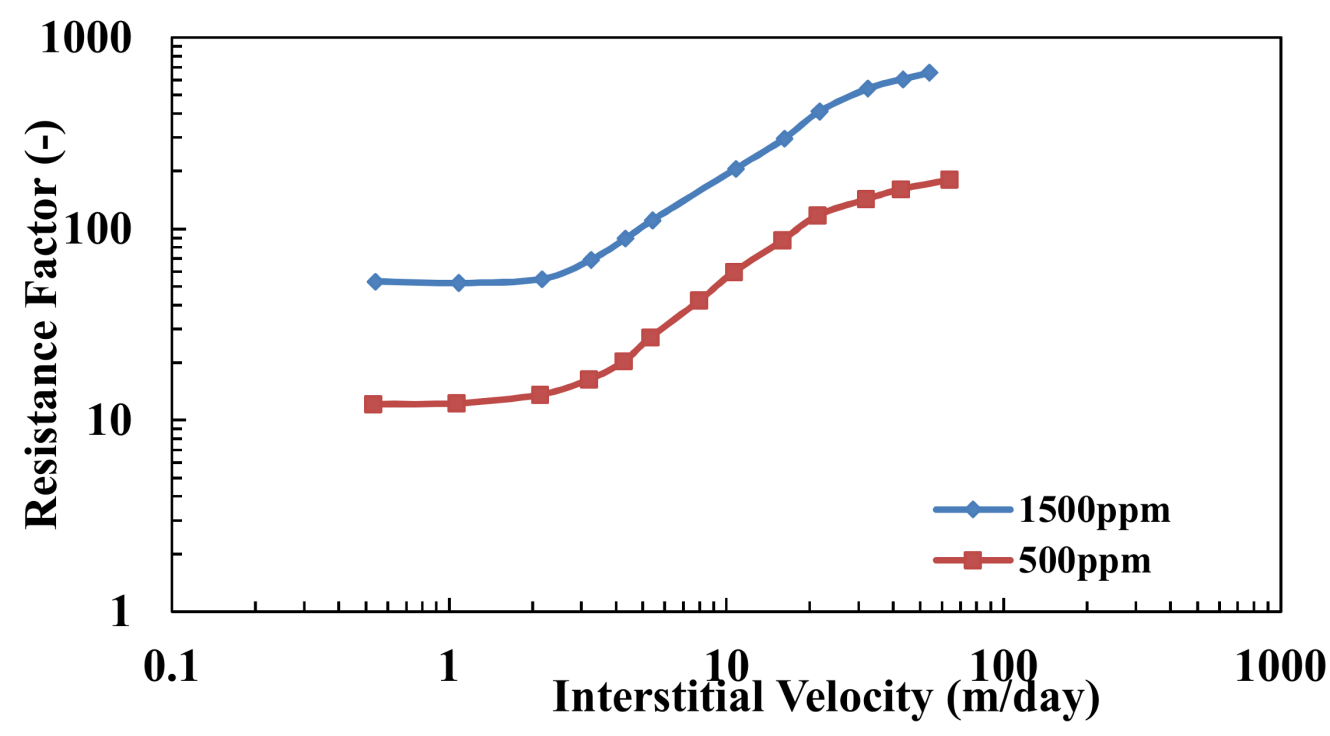

Figure 5. Resistance factor versus interstitial velocity of pre-filtered Flopaam 3630S HPAM polymer dissolved in $1 \mathrm{wt} \% \mathrm{NaCl}$ brine.

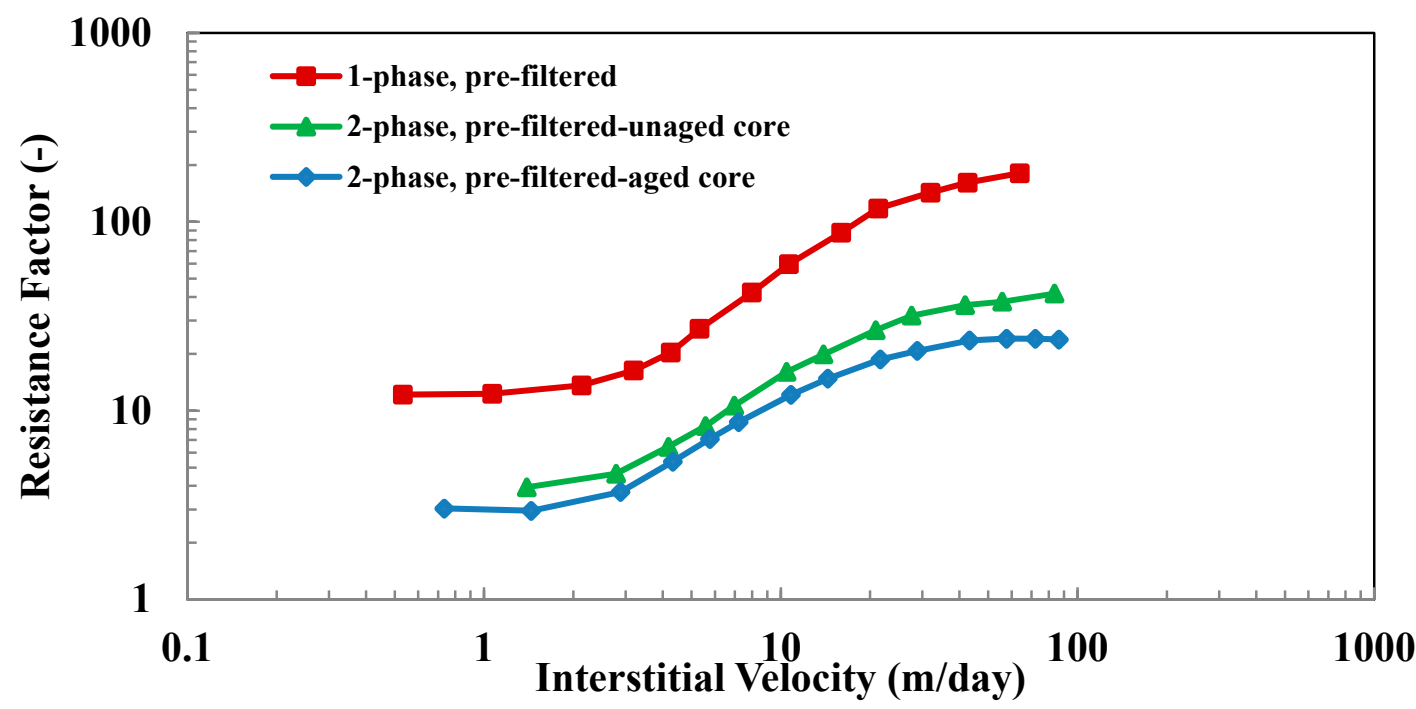

Figure 6. Resistance factor versus interstitial velocity of 500 ppm pre-filtered Flopaam 3630S partially hydrolyzed polyacrylamide (HPAM) polymer dissolved in $1 \mathrm{wt} \% \mathrm{NaCl}$ brine, single-phase polymer flow and polymer flow at residual oil saturation.

It is important to note that a lower resistance factor in the presence of oil is achieved while porous media is partially occupied by residual oil. Therefore, unlike the single-phase system, in which the pore volume (assuming no inaccessible pore volume) is available for polymer flow, only $\mathrm{PV}^{*}(1-\mathrm{Sor})$ is available for polymer flow in two-phase system. This influences and reduces permeability and therefore an even higher resistance factor is expected in the presence of oil. However, the results do not show such an effect, and a lower resistance factor and polymer injectivity is observed with the presence of oil in porous media, which supports the significance of the positive effect of oil on polymer injectivity. The effluent polymer viscosity is reduced by $18 \%$ compared to the injected polymer solution 
for the single-phase, water-wet case, while there is no reduction in effluent viscosity for the two-phase experiments (water-wet and non-water-wet). Shiran and Skauge [90] studied wettability using the same crude oil for aging and found that intermediate wettability was achieved. The end-point water relative permeability confirms that a similar condition was obtained.

Polymer injection in cores with residual oil results in a lower resistance factor which means better polymer injectivity. Furthermore, the resistance factor in the aged core with the non-water-wet state is lower than the resistance factor in the water-wet core. The lower resistance factor in the presence of oil could be attributed to lower adsorption/retention of polymer molecules on rock surface, as reported by Broseta et al. [10]. The rock surface in the presence of an oil film, and especially in less water-wet conditions is partially covered by crude oil polar components during flooding. Therefore, in comparison to single-phase systems, the rock surface has fewer adsorption sites to adsorb polymer molecules. The analysis of reduced apparent viscosity in the presence of oil, assumes that end-point water relative permeability remains constant for polymer as it does for water. The RRF measured with brine after the polymer injection is assumed constant for all rate variation of polymer flow. Under these assumptions a lower resistance factor and better polymer injectivity is expected.

\section{Polymer In-Situ Rheology in Radial Flow}

Recently, polymer injectivity was analyzed by matching field injectivity tests [5,6,91,92]. In addition to history matching, modification of equations to incorporate fractures and polymer degradation in the near-wellbore zone were reported. The laboratory experiment simplifies the analysis as additional complications like fractures and strong heterogeneity can be avoided.

In earlier studies of radial flow experiments, Skauge et al. [37] used local pressure taps as a function of radial distance from the injection well to derive in-situ rheology. These experiments demonstrated both shear thinning and strong apparent shear thickening behavior.

Two radial flow experiments were performed on circular Bentheimer sandstone disks of 1.6 and $2.6 \mathrm{D}$ permeability with $30 \mathrm{~cm}$ diameter and $2.2 \mathrm{~cm}$ thickness, see Table 7 . The first experiment was performed on a disk that was drained with crude oil and aged to non-water-wet conditions. The second experiment was performed in the absence of oil on a water-wet disk. For the first experiment, the disk was flooded extensively with brine to reach residual oil saturation, Sorw $=0.22$. Bump rates were applied to avoid oil mobilization by viscous forces during the subsequent polymer flood. The polymer flood was performed by first saturating the disk with polymer at a low rate to avoid mechanical degradation due to shearing. Thereafter, rate variations were performed to determine in-situ rheology of the polymer. A brine flush was performed between concentration slugs to remove non-adsorbed polymer.

Concentrations of 800 and $2000 \mathrm{ppm}$ were chosen to represent lower and upper boundaries of the semi-dilute region. The second experiment included the same steps, except for water flooding to Sorw. In this case, the water flood was performed to obtain a pressure reference for the subsequent polymer flood. No oil production was detected during polymer floods.

Differential pressure was measured by internal pressure ports located at different radii from the injection well. The 800-ppm HPAM solution was injected in the presence of residual oil at flow rates of 2.2 and $2.8 \mathrm{~mL} / \mathrm{min}$, and in the absence of residual oil at 2.0 and $4.0 \mathrm{~mL} / \mathrm{min}$. Differential pressure decay as a function of radial distance from injector is shown in Figure 7. The pressure transition zone from semi-steady-state to steady-state is extended compared to the case without oil. Most notable is the difference in pressure in the injection well. While differential pressures measured from internal pressure ports are higher for the two-phase system (as expected), well injection pressure is significantly lower in the presence of residual oil. Taking the pressure ratio of pressure ports at $\sim 1$ $\mathrm{cm}$ from injection well as a reference, injection pressure should be 5-6 times higher for the disk with oil, compared to the one without. Instead, the injection pressure is $25 \%$ lower. There may be several reasons for this observed result. One reason may be that the presence of oil reduces the effective pore volume, thereby leading to higher flow velocities for the polymer in the near-well region. This would 
subsequently lead to higher effective shear forces on the polymer, producing mechanical degradation. If mechanical degradation occurs, it has only a minor effect on the shear viscosity. The shear viscosity is $15.1 \mathrm{mPas}$ for the effluent sample taken at $2.0 \mathrm{~mL} / \mathrm{min}$, while it was $16.0 \mathrm{mPas}$ for the injected solution (measured at $22^{\circ} \mathrm{C}, 101 / \mathrm{s}$ ). However, as discussed in Section 2.2, it is the extensional viscosity that is the determining factor for high pressures in near-well region. Changes in extensional viscosity are intrinsically hard to measure and were not performed here. It is still possible that the increase in shear forces for the case with residual oil lead to a reduction in extensional viscosity but not for the case without oil where the effective pore volume was larger. The two other reasons are related to the wetting state of the porous media. If the oil is located in smaller pores, polymer flow is diverted to larger pores where it flows at higher velocities (higher flux). Since the velocity increase takes place in larger pores, only minor degradation would be expected. A third reason may be that porous media is fractionally oil-wet and that there is a difference in the slip conditions for the water-wet and the oil-wet surfaces. This may reduce effective shear for the oil-wet surfaces leading to reduced mechanical degradation. Although there have been speculations on the "lubricating" effect of oil-wet surfaces, no clear evidence of the effect on apparent viscosity or injectivity for core material have been shown to date. It is not possible to differentiate between the three phenomena based on the pressure data alone.

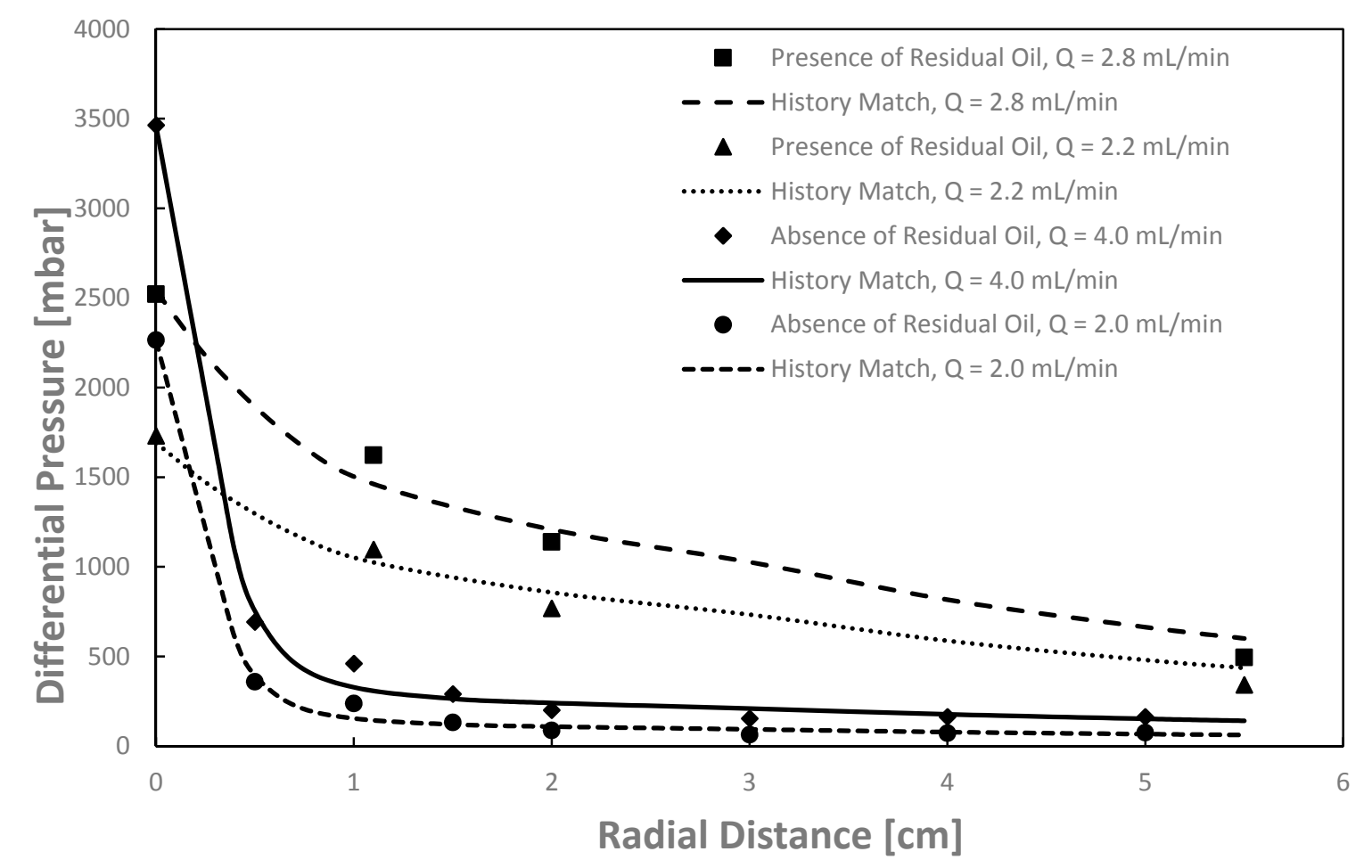

Figure 7. Differential pressure profiles for 800-ppm HPAM floods in the presence and absence of residual oil in radial geometry as a function of distance from injector to producer for four flow rates.

Each of the polymer floods were history matched using STARS (CMG). The measured differential pressures as a function of distance from injection well were used as history match parameters, while polymer apparent viscosity was used as a tuning variable. History matches and polymer rheology from both experiments for 800-ppm HPAM floods are shown in Figures 7 and 8, respectively. It is evident from Figure 8 that the polymer rheology is significantly influenced by the presence of residual oil. In terms of absolute values, the apparent viscosity is between a factor of 5 and 10 , and it is higher in the absence, compared to the presence of residual oil. Furthermore, the onset of apparent shear thickening shifts to lower velocities in the presence of residual oil. This occurrence is suggested to result from reduced propagation cross-section caused by the residual oil saturation. When flow 
channels in porous media become narrower, the extensional flow regime is reached at a lower flux, and HPAM exhibits viscoelastic behavior at an earlier stage, thus the onset of apparent shear thickening commences at a lower flux. The effect of shifting the onset of apparent shear thickening to a lower flux may be detrimental for injectivity. However, since the apparent shear thickening seems to be much more extensive in the absence of residual oil, the rheology shows that overall injectivity is significantly improved in presence of residual oil.

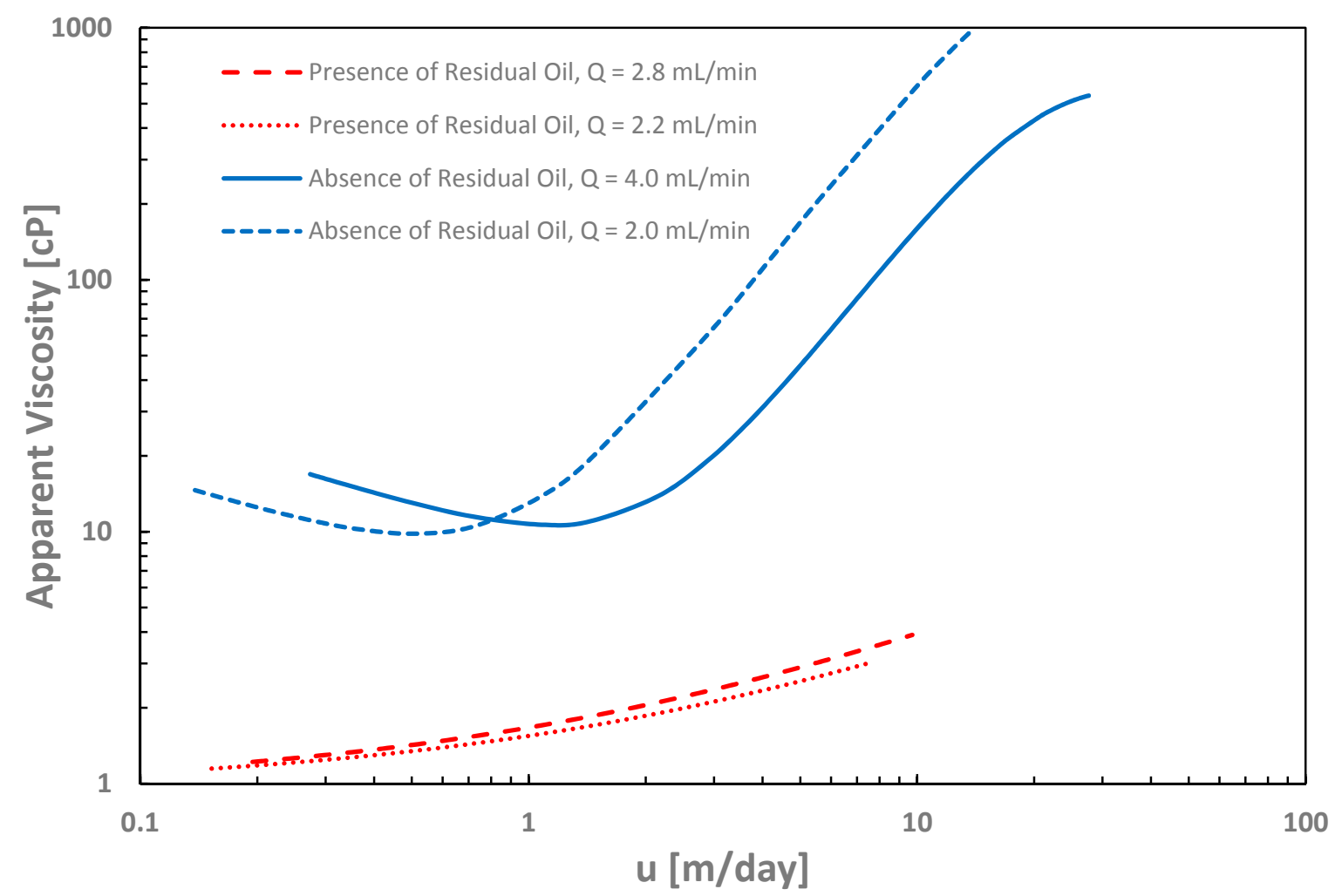

Figure 8. Apparent viscosity from history match of differential pressure for $800 \mathrm{ppm}$ HPAM in presence and absence of residual oil in radial geometry.

History matches and polymer rheology in the presence and absence of residual oil for $2000 \mathrm{ppm}$ floods are shown in Figures 9 and 10, respectively. In order to evaluate the influence of polymer concentration on in-situ rheology, 2000 ppm HPAM was injected in both disks. The differential pressures are shown in Figure 9. In this case, the injection rates were $Q=2.0$ and $5.0 \mathrm{~mL} / \mathrm{min}$ for the disk with no oil, and $\mathrm{Q}=1.4$ and $1.6 \mathrm{~mL} / \mathrm{min}$ for the disk with oil. These data show the same trend as for the 800 ppm injection: strong reduction in injection well pressure in the presence of residual oil and extension of the transition zone.

In accordance with the $800 \mathrm{ppm}$ solution, polymer viscosity was significantly higher in the absence compared to presence of residual oil, and ranged between a factor of 6 and 16 in their joint velocity interval, Figure 10. In addition, the $2000 \mathrm{ppm}$ solution also showed a decrease in the onset of apparent shear thickening in the presence of residual oil, consistent with the lower concentration solution investigated. Similar to the $800 \mathrm{ppm}$ solution, apparent shear thickening is observed to be much more extensive in absence of residual oil, thus improved injectivity in the presence of residual oil is further corroborated. 


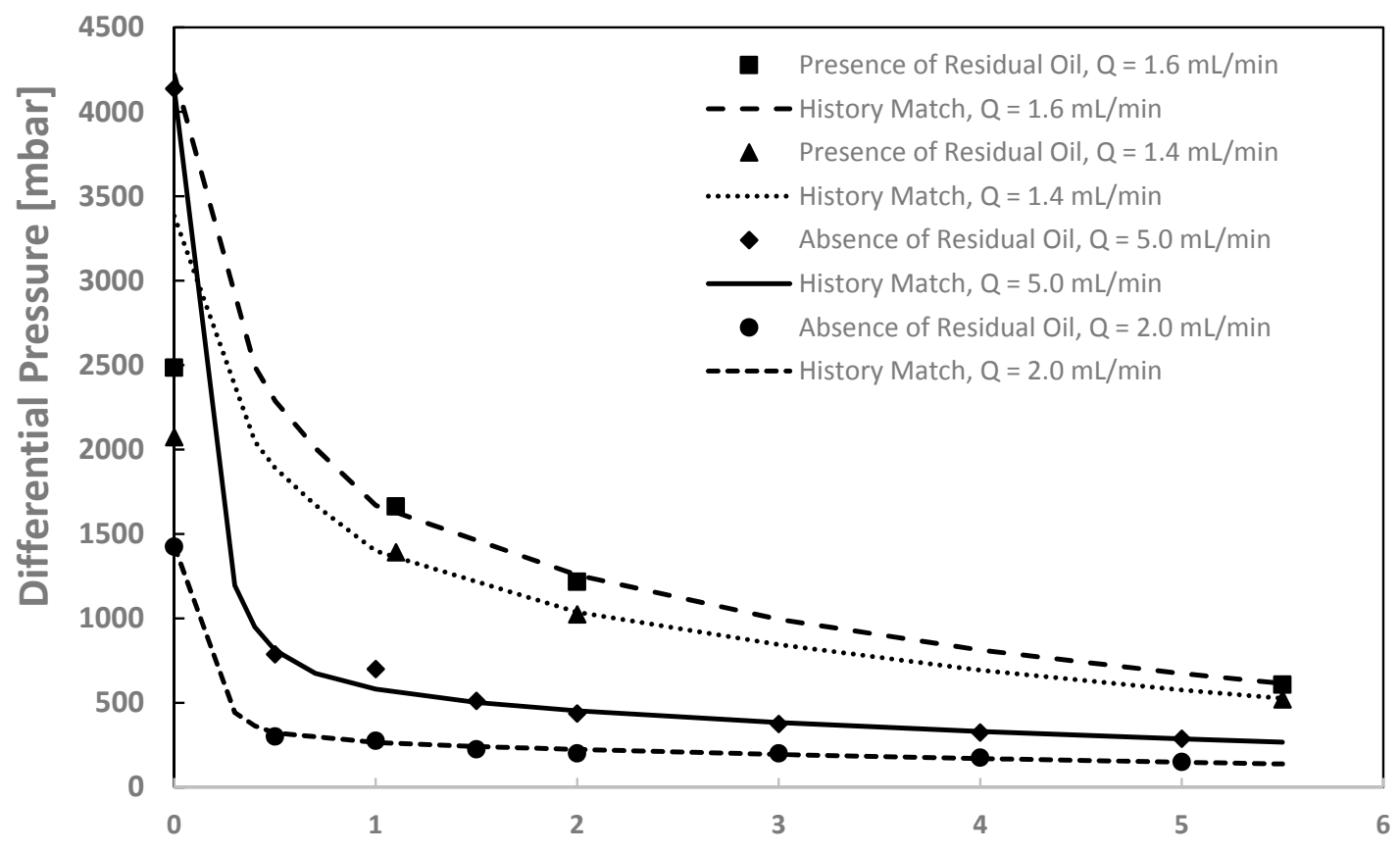

\section{Radial Distance [cm]}

Figure 9. Differential pressure profiles for 2000 ppm 3630 S HPAM floods in presence and absence of residual oil in radial geometry as a function of distance from injector to producer for four flow rates.

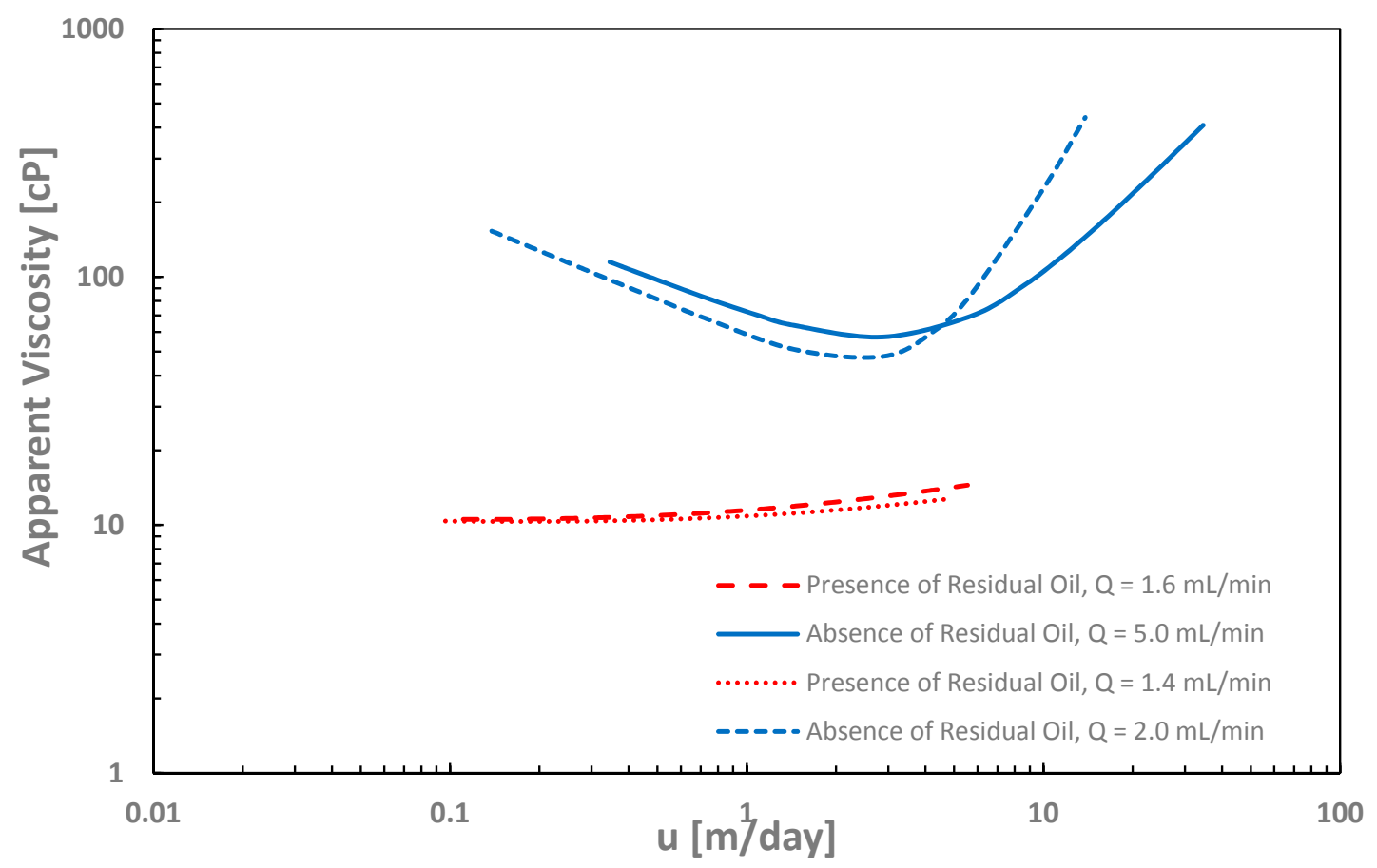

Figure 10. Apparent viscosity from the history match of differential pressure for 2000 ppm $3630 S$ HPAM in the presence and absence of residual oil in radial geometry. 


\section{Conclusions}

A review of polymer flow in a porous medium was presented. The available EOR analytical models we evaluated have limitations in accurately describing flow of polymer at high shear rates, e.g., near injector, and this leads to underestimating or overestimating of polymer injectivity.

The experimental results presented expand our insight into polymer flow in a porous medium. Shear thinning behavior may be present in core floods while bulk rheology is predominant from rheometer measurements. Linear polymer flow experiments are dominated by apparent shear thickening which is not measured in standard rheometers. The extensional viscosity, which is the main cause of the apparent shear thickening behavior, occurs at flow velocities strongly influenced by the porous media.

Linear core floods are commonly used for evaluating polymer in-situ rheology and injectivity, but they suffer from steady-state conditions throughout the core as opposed to the well injection situation where both pressure and shear forces are nonlinear gradients.

In the linear core floods, the onset of apparent shear thickening is independent of polymer concentration, when polymer type, brine composition and porous media are held constant. It is also independent of the presence of oil and wettability for the three cases evaluated here.

Radial flow injections show more complex in-situ rheology. The in-situ rheology shows a much higher degree of apparent shear thickening in the presence of oil. This may be due to restrictions in the pore space available. In the absence of oil, high concentration polymer (2000 ppm) showed shear thinning behavior. The onset of apparent shear thickening was shifted to higher flow velocities. There is a need for further development of numerical models that incorporate memory effects and possible kinetic effects for high polymer flow rates in the near-well region.

Both linear and radial experiments confirm lower apparent viscosity when oil is present in the porous medium. This conclusion is based on the assumption that brine end-point relative permeability is unchanged for polymer injection compared to two-phase flow by water injection. No extra oil was produced during polymer injection and this support the lowering of in-situ polymer viscosity in the presence of oil.

Author Contributions: A.S., B.S.S., and T.S. were responsible for the experimental program and simulation study. N.Z. structured the review contribution, while B.A.-S. and J.G.J. are Ph.D. students working on polymer injectivity, including the experimental study and simulation work. All authors contributed in writing the paper.

Funding: This research received no direct external funding.

Acknowledgments: The authors gratefully acknowledge support from the Norwegian Research Council, Petromaks 2 program. Badar Al-Shakry acknowledge support from, PDO, Petroleum Development Oman. Arne Skauge acknowledge support from Energi Simulations, Canada as the Energi Simulation EOR chair at University of Bergen.

Conflicts of Interest: The authors declare no conflicts of interest.

\section{Nomenclature}

$\begin{array}{ll}\mathrm{A} & \text { Cross section area } \\ \mathrm{C} & \text { Power-law constant } \\ \mathrm{C}_{\mathrm{p}} & \text { Polymer concentration } \\ D_{p} & \text { Grain size diameter } \\ \mathrm{De} & \text { Deborah number } \\ h & \text { Disk thickness } \\ \mathrm{H} & \text { Constant, equation } 11 \\ \mathrm{k} & \text { Constant, equation } 18 \\ \mathrm{~K}_{\mathrm{ei}} & \text { Effective permeability to polymer } \\ \mathrm{K} & \text { Permeability } \\ \mathrm{L} & \text { Length of model } \\ M_{w} & \text { Polymer molecular weight } \\ N_{1} & \text { Normal stress difference }\end{array}$


Ellis, Carreau or power-law constant

Pressure

Flow rate

Radius

Disk radius

Injection well radius

Resistance factor

RRF Residual resistance factor

$R_{e q} \quad$ Equivalent radius obtained from Blake-Kozeny model

$S_{w} \quad$ Water saturation

$\mathrm{T} \quad$ Temperature

Tr Trouton ratio

U Darcy velocity

Wi Weissenberg number

I Injectivity

$\alpha \quad$ Correction factor

$\beta \quad$ Constant, equation 10

$\omega$ Angular velocity

$\dot{\varepsilon} \quad$ Stretch rate

$\Delta P \quad$ Pressure drop

$\dot{\gamma}$ Shear rate

$\tau_{1 / 2} \quad$ Ellis model parameter

$\dot{\gamma}_{\text {eff }} \quad$ Effective shear rate

$\dot{\gamma}_{a p p} \quad$ Apparent shear rate

$\dot{\gamma}_{c} \quad$ Critical shear rate

$\lambda \quad$ Polymer relaxation time

$\lambda_{z} \quad$ Zimm relaxation time

$\mu \quad$ Viscosity

$\mu_{\text {app }} \quad$ Apparent viscosity

$\mu_{e f f} \quad$ Effective viscosity

$\mu_{0} \quad$ Upper Newtonian plateau

$\mu_{s} \quad$ Solvent viscosity

$\mu_{\text {sh }} \quad$ Shear rate viscosity

$\mu_{e} \quad$ Elongational viscosity

$\mu_{\infty} \quad$ Lower Newtonian plateau

$\phi \quad$ Porosity

$\psi \quad$ Tortuosity

$\theta_{f} \quad$ Characteristic relaxation time of fluid

$\theta_{p} \quad$ Characteristic time of porous media

\section{References}

1. Seright, R.S. The Effects of Mechanical Degradation and Viscoelastic Behavior on Injectivity of Polyacrylamide Solutions. Soc. Pet. Eng. J. 1983, 23, 475-485. [CrossRef]

2. Shuler, P.J.; Kuehne, D.L.; Uhl, J.T.; Walkup, G.W., Jr. Improving Polymer Injectivity at West Coyote Field, California. Soc. Pet. Eng. Reserv. Eng. 1987, 2, 271-280. [CrossRef]

3. Southwick, J.G.; Manke, C.W. Molecular Degradation, Injectivity, and Elastic Properties of Polymer Solutions. Soc. Pet. Eng. Reserv. Eng. 1988, 3, 1193-1201. [CrossRef]

4. Yerramilli, S.S.; Zitha, P.L.J.; Yerramilli, R.C. Novel Insight into Polymer Injectivity for Polymer Flooding. Presented at the SPE European Formation Damage Conference and Exhibition, Noordwijk, The Netherlands, 5-7 June 2013. [CrossRef]

5. Lotfollahi, M.; Farajzadeh, R.; Delshad, M.; Al-Abri, K.; Wassing, B.M.; Mjeni, R.; Awan, K.; Bedrikovetsky, P. Mechanistic Simulation of Polymer Injectivity in Field Tests. Presented at the SPE Enhanced Oil Recovery Conference, Kuala Lumpur, Malaysia, 11-13 August 2015. [CrossRef]

6. Glasbergen, G.; Wever, D.; Keijzer, E.; Farajzadeh, R. Injectivity Loss in Polymer Floods: Causes, Preventions and Mitigations. Presented at the SPE Kuwait Oil \& Gas Show and Conference, Mishref, Kuwait, 11-14 October 2015. [CrossRef] 
7. Al-Shakry, B.; Shiran, B.S.; Skauge, T.; Skauge, A. Enhanced Oil Recovery by Polymer Flooding: Optimizing Polymer Injectivity. Presented at the SPE Kingdom of Saudi Arabia Technical Symposium and Exhibition, Dammam, Saudi Arabia, 23-26 April 2018.

8. Skauge, T.; Kvilhaug, O.A.; Skauge, A. Influence of Polymer Structural Conformation and Phase Behaviour on In-situ Viscosity. Presented at the 18th European Symposium on Improved Oil Recovery, Dresden, Germany, 14-16 April 2015. [CrossRef]

9. Hughes, D.S.; Teeuw, D.; Cottrell, C.W.; Tollas, J.M. Appraisal of the Use of Polymer Injection To Suppress Aquifer Influx and To Improve Volumetric Sweep in a Viscous Oil Reservoir. Soc. Pet. Eng. 1990, 5, 33-40. [CrossRef]

10. Broseta, D.; Medjahed, F.; Lecourtier, J.; Robin, M. Polymer Adsorption/Retention in Porous Media: Effects of Core Wettability on Residual Oil. Soc. Pet. Eng. 1995, 3, 103-112. [CrossRef]

11. Sorbie, K.S. Polymer-Improved Oil Recovery; Blackie and Son Ltd.: Glasgow, UK, 1991.

12. Lake, L.W. Enhanced Oil Recovery; Prentice Hall: Upper Saddle River, NJ, USA, 1989.

13. Savins, J.G. Non-Newtonian Flow through Porous Media. Ind. Eng. Chem. 1969, 61, 18-47. [CrossRef]

14. Sadowski, T.J. Non-Newtonian Flow through Porous Media. II. Experimental. J. Rheol. 1965, 9, $251-271$. [CrossRef]

15. Sochi, T. Non-Newtonian flow in porous media. Polymer 2010, 51, 5007-5023. [CrossRef]

16. Sadowski, T.J.; Bird, R.B. Non-Newtonian Flow through Porous Media. I. J. Rheol. 1965, 9, 243-250. [CrossRef]

17. Teeuw, D.; Hesselink, F.T. Power-Law Flow And Hydrodynamic Behaviour of Biopolymer Solutions In Porous Media. Presented at the SPE Fifth International Symposium on Oilfield and Geothermal Chemistry, Stanford, CA, USA, 28-30 May 1980. [CrossRef]

18. Cannella, W.J.; Huh, C.; Seright, R.S. Prediction of Xanthan Rheology in Porous Media. Presented at the 63rd Annual Technical Conference and Exhibition of the Society of Petroleum Engineers, Houston, TX, USA, 2-5 October 1988. [CrossRef]

19. Fletcher, A.J.P.; Flew, S.R.G.; Lamb, S.P.; Lund, T.; Bjornestad, E.; Stavland, A.; Gjovikli, N.B. Measurements of Polysaccharide Polymer Properties in Porous Media. Prepared for presentation at the SPE International Symposium on Oilfield Chemistry, Anaheim, CA, USA, 20-22 February 1991. [CrossRef]

20. Sorbie, K.S.; Clifford, P.J.; Jones, E.R.W. The Rheology of Pseudoplastic Fluids in Porous Media Using Network Modeling. J. Colloid Interface Sci. 1989, 130, 508-534. [CrossRef]

21. Lopez, X.; Valvatne, P.H.; Blunt, M.J. Predictive network modeling of single-phase non-Newtonian flow in porous media. J. Colloid Interface Sci. 2003, 264, 256-265. [CrossRef]

22. Pearson, J.R.A.; Tardy, P.M.J. Models for flow of non-Newtonian and complex fluids through porous media. J. Non-Newton. Fluid Mech. 2002, 102, 447-473. [CrossRef]

23. Willhite, G.P.; Uhl, J.T. Correlation of the Flow of Flocon 4800 Biopolymer with Polymer Concentration and Rock Properties in Berea Sandstone. In Water-Soluble Polymers for Petroleum Recovery; Springer Publishing: Manhattan, NY, USA, 1988.

24. Bird, R.B.; Stewart, W.E.; Lightfoot, E.N. Transport Phenomena; John Wiley and Sons, Inc.: New York, NY, USA, 1960.

25. Christopher, R.H.; Middleman, S. Power-Law Flow through a Packed Tube. Ind. Eng. Chem. Fundam. 1965, 4, 422-426. [CrossRef]

26. Hirasaki, G.J; Pope, G.A. Analysis of Factors Influencing Mobility and Adsorption in the Flow of Polymer Solution Through Porous Media. Soc. Pet. Eng. J. 1974, 14, 337-346. [CrossRef]

27. Duda, J.L.; Hong, S.-A.; Klaus, E.E. Flow of Polymer-Solutions in Porous-Media-Inadequacy of the Capillary Model. Ind. Eng. Chem. Fundam. 1983, 22, 299-305. [CrossRef]

28. Kishbaugh, A.J.; McHugh, A.J. A rheo-optical study of shear-thickening and structure formation in polymer solutions. Part I: Experimental. Rheol. Acta 1993, 32, 9-24. [CrossRef]

29. Pope, D.P.; Keller, A. Alignment of Macromolecules in Solution by Elongational Flow-Study of Effect of Pure Shear in a 4 Roll Mill. Colloid Polym. Sci. 1977, 255, 633-643. [CrossRef]

30. Binding, D.M.; Jones, D.M.; Walters, K. The Shear and Extensional Flow Properties of M1. J. Non-Newton. Fluid Mech. 1990, 35, 121-135. [CrossRef]

31. Gogarty, W.B. Rheological Properties of Pseudoplastic Fluids in Porous Media. Soc. Pet. Eng. J. 1967, 7, 149-160. [CrossRef] 
32. Perrin, C.L.; Tardy, P.M.J.; Sorbie, K.S.; Crawshaw, J.C. Experimental and modeling study of Newtonian and non-Newtonian fluid flow in pore network micromodels. J. Colloid Interface Sci. 2006, 295, 542-550. [CrossRef] [PubMed]

33. Sochi, T.; Blunt, M.J. Pore-scale network modeling of Ellis and Herschel-Bulkley fluids. J. Pet. Sci. Eng. 2008, 60, 105-124. [CrossRef]

34. Balhoff, M.T.; Thompson, K.E. A macroscopic model for shear-thinning flow in packed beds based on network modeling. Chem. Eng. Sci. 2006, 61, 698-719. [CrossRef]

35. Zamani, N.; Bondino, I.; Kaufmann, R.; Skauge, A. Computation of polymer in-situ rheology using direct numerical simulation. J. Pet. Sci. Eng. 2017, 159, 92-102. [CrossRef]

36. Hejri, S.; Willhite, G.P.; Green, D.W. Development of Correlations to Predict Biopolymer Mobility in Porous Media. SPE Reserv. Eng. 1991, 6, 91-98. [CrossRef]

37. Skauge, T.; Skauge, A.; Salmo, I.C.; Ormehaug, P.A.; Al-Azri, N.; Wassing, L.M.; Glasbergen, G.; Van Wunnik, J.N.; Masalmeh, S.K. Radial and Linear Polymer Flow-Influence on Injectivity. Prepared for presentation at the SPE Improved Oil Recovery Conference, Tulsa, Oklahoma, 11-13 April 2016. [CrossRef]

38. Faber, T.E. Fluid Dynamics for Physicists; Cambridge University Press: Cambridge, UK, 1995.

39. Brown, E.; Jaeger, H.M. The role of dilation and confining stresses in shear thickening of dense suspensions. J. Rheol. 2012, 56, 875-923. [CrossRef]

40. Choplin, L.; Sabatie, J. Threshold-Type Shear-Thickening in Polymeric Solutions. Rheol. Acta 1986, 25, 570-579. [CrossRef]

41. Indei, T.; Koga, T.; Tanaka, F. Theory of shear-thickening in transient networks of associating polymer. Macromol. Rapid Commun. 2005, 26, 701-706. [CrossRef]

42. Odell, J.A.; Müller, A.J.; Keller, A. Non-Newtonian behaviour of hydrolysed polyacrylamide in strong elongational flows: A transient network approach. Polymer 1988, 29, 1179-1190. [CrossRef]

43. Degennes, P.G. Coil-Stretch Transition of Dilute Flexible Polymers under Ultrahigh Velocity-Gradients. J. Chem. Phys. 1974, 60, 5030-5042. [CrossRef]

44. Trouton, F.T. On the coefficient of viscous traction and its relation to that of viscosity. R. Soc. 1906, 77, 426-440. [CrossRef]

45. Edwards, B.J.; Keffer, D.I.; Reneau, C.W. An examination of the shear-thickening behavior of high molecular weight polymers dissolved in low-viscosity newtonian solvents. J. Appl. Polym. Sci. 2002, 85, 1714-1735. [CrossRef]

46. Hatzignatiou, D.G.; Moradi, H.; Stavland, A. Experimental Investigation of Polymer Flow through Waterand Oil-Wet Berea Sandstone Core Samples. Presented at the EAGE Annual Conference \& Exhibition incorporationg SPE Europec, London, UK, 10-13 June 2013. [CrossRef]

47. McKinley, G.H.; Sridhar, T. Filament-stretching rheometry of complex fluids. Annu. Rev. Fluid Mech. 2002, 34, 375-415. [CrossRef]

48. Fuller, G.G.; Cathey, C.A.; Hubbard, B.; Zebrowski, B.E. Extensional Viscosity Measurements for Low-Viscosity Fluids. J. Rheol. 1987, 31, 235-249. [CrossRef]

49. Meadows, J.; Williams, P.A.; Kennedy, J.C. Comparison of the Extensional and Shear Viscosity Characteristics of Aqueous Hydroxyethylcellulose Solutions. Macromolecules 1995, 28, 2683-2692. [CrossRef]

50. Anna, S.L.; McKinley, G.H.; Nguyen, D.A.; Sridhar, T.; Muller, S.J.; Huang, J.; James, J.F. An interlaboratory comparison of measurements from filament-stretching rheometers using common test fluids. J. Rheol. 2001, 45, 83-114. [CrossRef]

51. Shipman, R.W.G.; Denn, M.M.; Keunings, R. Mechanics of the Falling Plate Extensional Rheometer. J. Non-Newton. Fluid Mech. 1991, 40, 281-288. [CrossRef]

52. Sridhar, T.; Tirtaatmadja, V.; Nguyen, D.A.; Gupta, R.K. Measurement of Extensional Viscosity of Polymer-Solutions. J. Non-Newton. Fluid Mech. 1991, 40, 271-280. [CrossRef]

53. Tirtaatmadja, V.; Sridhar, T. A Filament Stretching Device for Measurement of Extensional Viscosity. J. Rheol. 1993, 37, 1081-1102. [CrossRef]

54. James, D.F.; Chandler, G.M.; Armour, S.J. A Converging Channel Rheometer for the Measurement of Extensional Viscosity. J. Non-Newton. Fluid Mech. 1990, 35, 421-443. [CrossRef] 
55. Chauveteau, G. Moluecular interpretation of several different properties of flow of coiled polymer solutions through porous media in oil recovery conditions. Presented at the 56th Annual Fall Technical Conference and Exhibition of the society of Petroleum Engineers of AIME, San Antonio, TX, USA, 5-7 October 1981. [CrossRef]

56. Lewandowska, K. Comparative studies of rheological properties of polyacrylamide and partially hydrolyzed polyacrylamide solutions. J. Appl. Polym. Sci. 2007, 103, 2235-2241. [CrossRef]

57. Briscoe, B.; Luckham, P.; Zhu, S.P. Pressure influences upon shear thickening of poly(acrylamide) solutions. Rheol. Acta 1999, 38, 224-234. [CrossRef]

58. Dupuis, D.; Lewandowski, F.Y.; Steiert, P.; Wolff, C. Shear Thickening and Time-Dependent Phenomena-The Case of Polyacrylamide Solutions. J. Non-Newton. Fluid Mech. 1994, 54, 11-32. [CrossRef]

59. Jiang, B.; Keffer, D.J.; Edwards, B.J.; Allred, J.N. Modeling shear thickening in dilute polymer solutions: Temperature, concentration, and molecular weight dependencies. J. Appl. Polym. Sci. 2003, 90, 2997-3011. [CrossRef]

60. Clarke, A.; Howe, A.M.; Mitchell, J.; Staniland, J.; Hawkes, L.A. How Viscoelastic-Polymer Flooding Enhances Displacement Efficiency. Soc. Pet. Eng. J. 2016, 21, 675-687. [CrossRef]

61. Delshad, M.; Kim, D.H.; Magbagbeola, O.A.; Huh, C.; Pope, G.A.; Tarahhom, F. Mechanistic Interpretation and Utilization of Viscoelastic Behavior of Polymer Solutions for Improved Polymer-Flood Efficiency. Prepared for presentation at the 2008 SPE/DOE Improved Oil Recovery Symposium, Tulsa, OK, USA, 19-23 April 2008. [CrossRef]

62. Stavland., A.; Jonsbråten, H.C.; Lohne, A.; Moen, A.; Giske, N.H. Stavland. A.; Jonsbråten, H.C.; Lohne, A.; Moen, A.; Giske, N.H. Polymer Flooding-Flow Properties in Porous Media Versus Rheological Parameters. Presented at the SPE EUROPEC/EAGE Annual Conference and Exhibition, Barcelona, Spain, 14-17 June 2010. [CrossRef]

63. Aitkadi, A.; Carreau, P.J.; Chauveteau, G. Rheological Properties of Partially Hydrolyzed Polyacrylamide Solutions. J. Rheol. 1987, 31, 537-561. [CrossRef]

64. Lee, K.; Huh, C.; Sharma, M.M. Impact of Fractures Growth on Well Injectivity and Reservoir Sweep during Waterflood and Chemical EOR Processes. Presented at the SPE Annual Technical Conference and Exhibition, Denver, CO, USA, 30 October-2 November 2011. [CrossRef]

65. Hu, Y.; Wang, S.Q.; Jamieson, A.M. Rheological and Rheooptical Studies of Shear-Thickening Polyacrylamide Solutions. Macromolecules 1995, 28, 1847-1853. [CrossRef]

66. Cho, Y.H.; Dan, K.S.; Kim, B.C. Effects of dissolution temperature on the rheological properties of polyvinyl alchol solutions in dimethyl sulfoxide. Korea-Aust. Rheol. J. 2008, 20, 73-77.

67. Zamani, N.; Bondino, I.; Kaufmann, R.; Skauge, A. Effect of porous media properties on the onset of polymer extensional viscosity. J. Pet. Sci. Eng. 2015, 133, 483-495. [CrossRef]

68. Gupta, R.K.; Sridhar, T. Viscoelastic Effects in Non-Newtonian Flows through Porous-Media. Rheol. Acta 1985, 24, 148-151. [CrossRef]

69. Smith, F.W. Behavior of Partially Hydrolyzed Polyacrylamide Solutions in Porous Media. J. Pet. Technol. 1970, 22, 148-156. [CrossRef]

70. Kemblowski, Z.; Dziubinski, M. Resistance to Flow of Molten Polymers through Granular Beds. Rheol. Acta 1978, 17, 176-187. [CrossRef]

71. Wissler, E.H. Viscoelastic Effects in the Flow of Non-Newtonian Fluids through a Porous Medium. Ind. Eng. Chem. Fundam. 1971, 10, 411-417. [CrossRef]

72. Vossoughi, S.; Seyer, F.A. Pressure-Drop for Flow of Polymer-Solution in a Model Porous-Medium. Can. J. Chem. Eng. 1974, 52, 666-669. [CrossRef]

73. Marshall, R.J.; Metzner, A.B. Flow of Viscoelastic Fluids through Porous Media. Ind. Eng. Chem. Fundam. 1967, 6, 393-400. [CrossRef]

74. Durst, F.; Haas, R.; Interthal, W. Laminar and Turbulent Flows of Dilute Polymer-Solutions-A Physical Model. Rheol. Acta 1982, 21, 572-577. [CrossRef]

75. Heemskerk, J.; Rosmalen, R.; Janssen-van, R.; Holtslag, R.J.; Teeuw, D. Quantification of Viscoelastic Effects of Polyacrylamide Solutions. Presented at the SPE/DOE Fourth Symposium on Enhanced Oil Recovery, Tulsa, OK, USA, 15-18 April 1984. [CrossRef] 
76. Metzner, A.B.; White, J.L.; Denn, M.M. Constitutive equations for viscoelastic fluids for short deformation periods and for rapidly changing flows: Significance of the deborah number. AIChE J. 1966, 12, 863-866. [CrossRef]

77. Masuda, Y.; Tang, K.-C.; Miyazawa, M.; Tanaka, S. 1D Simulation of Polymer Flooding Including the Viscoelastic Effect of Polymer Solution. SPE Reverv. Eng. 1992, 7, 247-252. [CrossRef]

78. Haas, R.; Durst, F. Viscoelastic Flow of Dilute Polymer-Solutions in Regularly Packed-Beds. Rheol. Acta 1982, 21, 566-571. [CrossRef]

79. Garrouch, A.A.; Gharbi, R.C. A Novel Model for Viscoelastic Fluid Flow in Porous Media. Presented at the 2006 SPE Annual Technical Conference and Exhibition, San Antonio, TX, USA, 24-27 September 2006. [CrossRef]

80. Ranjbar, M.; Rupp, J.; Pusch, G.; Meyn, R. Quantification and Optimization of Viscoelastic Effects of Polymer Solutions for Enhanced Oil Recovery. Presented at the SPE/DOE Eight Symposium on Enhanced Oil Recovery, Tulsa, OK, USA, 22-24 April 1992. [CrossRef]

81. Kawale, D.; Marques, E.; Zitha, P.L.J.; Kreutzer, M.T.; Rossen, W.R.; Boukany, P.E. Elastic instabilities during the flow of hydrolyzed polyacrylamide solution in porous media: effect of pore-shape and salt. Soft Matter 2017, 13, 765-775. [CrossRef] [PubMed]

82. Seright, R.S.; Seheult, J.M.; Talashek, T. Injectivity Characteristics of EOR Polymers. SPE Reserv. Eval. Eng. 2009, 12, 783-792. [CrossRef]

83. Kulawardana, E.U.; Koh, H.; Kim, D.H.; Liyanage, P.J.; Upamali, K.; Huh, C.; Weerasooriya, U.; Pope, G.A. Rheology and Transport of Improved EOR Polymers under Harsh Reservoir Conditions. Presented at the Eighteenth SPE Improved Oil Recovery Symposium, Tulsa, OK, USA, 14-18 April 2012. [CrossRef]

84. Sharma, A.; Delshad, M.; Huh, C.; Pope, G.A. A Practical Method to Calculate Polymer Viscosity Accurately in Numerical Reservoir Simulators. Presented at the SPE Annual Technical Conference and Exhibition, Denver, CO, USA, 31 October-2 November 2011. [CrossRef]

85. Manichand, R.N.; Moe Soe Let, K.P.; Gil, L.; Quillien, B.; Seright, R.S. Effective Propagation of HPAM Solutions Through the Tambaredjo Reservoir During a Polymer Flood. SPE Prod. Oper. 2013, 28, 358-368. [CrossRef]

86. Zaitoun, A.; Makakou, P.; Blin, N.; Al-Maamari, R.S.; Al-Hashmi, A.-A.R.; Abdel-Goad, M.; Al-Sharji, H.H. Shear Stability of EOR Polymers. SPE J. 2011, 17, 335-339. [CrossRef]

87. Suri, A.; Sharma, M.M.; Peters, E. Estimates of Fracture Lengths in an Injection Well by History Matching Bottomhole Pressures and Injection Profile. SPE Reserv. Eval. Eng. 2011, 14, 405-417. [CrossRef]

88. Zechner, M.; Clemens, T.; Suri, A.; Sharma, M.M. Simulation of Polymer Injection under Fracturing Conditions-A Field Pilot in the Matzen Field, Austria. SPE Reserv. Eval. Eng. 2014, 18, 236-249. [CrossRef]

89. Van den Hoek, P.; Mahani, H.; Sorop, T.; Brooks, D.; Zwaan, M.; Sen, S.; Shuaili, K.; Saadi, F. Application of Injection Fall-Off Analysis in Polymer flooding. Presented at the 74th EAGE Conference \& Exhibition incorporating SPE EUROPEC 2012, Copenhagen, Denmark, 4-7 June 2012. [CrossRef]

90. Shiran, B.S.; Skauge, A. Wettability and Oil Recovery by Polymer and Polymer Particles. Presented at the SPE Asia Pacific Enhanced Oil Recovery Conference, Kuala Lumpur, Malaysia, 11-13 August 2015. [CrossRef]

91. Al-Abri, K.; Al-Mjeni, R.; Al-Bulushi, N.K.; Awan, K.; Al-Azri, N.; Al-Riyami, O.; Al-Rajhi, S.; Teeuwisse, S.; Ghulam, J.; Abu-Shiekha, I.; et al. Reducing Key Uncertainities Prior to a Polymer Injection Trial in a Heavy Oil Reservoir in Oman. Presented at SPE EOR Conference at OGWA, Muscat, Oman, 31 March-2 April 2014. [CrossRef]

92. Li, Z.; Delshad, M. Development of an Analytical Injectivity Model for Non-Newtonian Polymer Solutions. Presented at the SPE Reservoir Simulation Symposium, Woodlands, TX, USA, 18-20 February 2014. [CrossRef]

(C) 2018 by the authors. Licensee MDPI, Basel, Switzerland. This article is an open access article distributed under the terms and conditions of the Creative Commons Attribution (CC BY) license (http:/ / creativecommons.org/licenses/by/4.0/). 